\title{
Concurrent Dual-Band Modeling and Digital Predistortion in the Presence of Unfilterable Harmonic Signal Interference
}

\author{
Meenakshi Rawat, Member, IEEE, Patrick Roblin, Senior Member, IEEE, \\ Christophe Quindroit, Khan Salam, Member, IEEE, and Chenggang Xie, Member, IEEE
}

\begin{abstract}
Motivated by advanced-generation signals and corresponding trends for multiband, broadband, and ultra-wideband transmitters, several models have recently been proposed for digital predistortion in a dual-band concurrent transmission. These state-of-the-art models assume that two frequencies of operation are uncorrelated and harmonic products can be filtered out. However, when the harmonic of one signal falls on the frequency band of another signal, it cannot be removed with filters. This paper proposes a 3-D harmonic memory polynomial based model for the dual-band concurrent transmission in the presence of harmonic interferences. The model is extracted and predistortion is implemented using a low-cost field-programmable gate-array-based system including a transmitter and a feedback receiver. Using the proposed model, a performance improvement up to $22 \mathrm{~dB}$ in terms of normalized mean square error and performance improvement up to $20 \mathrm{~dB}$ in terms of the adjacent channel power ratio is achieved compared to a conventional dual-band memory polynomial model not including harmonics.
\end{abstract}

Index Terms-Digital predistortion (DPD), dual band, harmonic, modeling, power amplifiers (PAs).

\section{INTRODUCTION}

D UE TO the ever-increasing number of data users, there is a strong motivation in developing multiband and wideband devices for transmitters and receivers to accommodate multiple frequencies in one transceiver system. The multiband and multi-standard radio architectures [1], [2] also allow for seamless transition from the older standard [e.g., third generation $(3 \mathrm{G})$ ] to new standards [e.g., fourth generation $(4 \mathrm{G})$ ] with backward compatibility. This has motivated the deployment of

Manuscript received September 01, 2014; revised November 12, 2014; accepted December 21, 2014. Date of publication January 20, 2015; date of current version February 03, 2015. This work was supported by Rockwell Collins under a project grant. This work was supported in part by the National Science Foundation (NSF) Collaborative under Grant ECS-1129013.

M. Rawat is with the Department of Electronics and Communications Engineering, Indian Institute of Technology, Roorkee 247667, India (e-mail: meenakshirawat@ieee.org).

P. Roblin is with the Department of Electrical and Computer Engineering, The Ohio State University, Columbus, OH 43210 USA (e-mail: roblin.1@osu. edu).

C. Quindroit was with the Department of Electrical and Computer Engineering, The Ohio State University, Columbus, OH 43210 USA. He is now with LPA Concepts, 33650 Martillac, France.

K. Salam and C. Xie are with the Advanced Technology Center, Rockwell Collins, Cedar Rapids, IA 52498 USA.

Color versions of one or more of the figures in this paper are available online at http://ieeexplore.ieee.org.

Digital Object Identifier 10.1109/TMTT.2014.2387289 commercial dual-band base stations. Moreover, the upcoming generation of wireless communication signals [e.g., fifth generation $(5 \mathrm{G})]$, which are promoting schemes such as carrier aggregation techniques [3], requiring concurrent transmission of signals at more than one frequency band, has renewed interest in the design of dual-band [4], [5] and broadband [6] power amplifiers (PAs).

The concurrent operation of such dual-band PAs with envelope exhibiting high PAPR signals gives rise to new in-band and out-of-band nonlinear distortions when the different nonlinear cross-products of the two signals interact with the bands of interest [7]-[21]. Therefore, within the past five years, linearization of concurrent multiband PA has been a topic of interest in software-defined radio (SDR) and digital predistortion (DPD) research communities. In [7], a concurrent dual-band predistortion technique at IF is conducted in a simulated environment. However, when two bands are widely spaced, together they create a very broadband signal, which imposes stringent requirements on high-sampling-rate digital-to-analog converters (DACs) and analog-to-digital converters (ADCs). As most of the frequency range between the two bands is not used for transmission, this requirement for costly high-speed DACs and ADCs is spectrally inefficient and impractical. In [8]-[10], a frequency-selective algorithm is proposed to provide a digital solution, which allows processing of each band individually eventually allowing for the use of two medium speed DACs and ADCs in each path. However, the original real-time linearization setup did not include demodulation capability in the observation path, and the predistorter coefficients in the field programmable gate array (FPGA) were tuned based on spectrum observations. In [11] and [12], the frequency-selective method was demonstrated for a three-band excitation.

In [13] and [14], a feedback receiver (vector signal analyzer) is employed to access the demodulated concurrent PA outputs and the frequency-selective linearization concept of [8] is extended to include time-selective memory effects, and is denoted as 2-D digital predistortion (2-D DPD).

Other research efforts has been focused towards improving stability, performance, and reducing application and algorithm complexity of the 2-D-DPD algorithm. A cubic-spline-based model is proposed to replace polynomials in 2-D DPD due to its better extrapolation properties in [15]. A rational-functionbased two-step approach for better modeling performance is proposed in [16]. A variable-memory-depth lookup-table-based 
implementation is proposed in [17]. A subsampling solution for feedback loop is proposed in [18] to simplify and reduce the complexity of the dual-band linearization architecture, which requires only one observation path for both bands. A 2-D augmented Hammerstein (2-D-AH) model [19] and 2-D modified memory polynomial (2-D-MMP) model [20] are proposed to reduce the complexity of the 2-D-DPD model. Similarly, in [21], a pruning method is applied to 2-D DPD with the aim to reduce the needed number of coefficient while achieving similar distortion cancellation results. To provide better numerical stability to the solution of 2-D DPD, 2-D orthogonal memory polynomials are introduced in [22], which also discusses time multiplexing method to reduce the implementation complexity in a FPGA testbed.

Some other research attempts have focused on the joint mitigation of modulator imbalance along with distortion arising from nonlinear concurrent dual-band operation. In [23], realvalued neural networks are used as such a solution, while [24] and [25] used dual-input truncated Volterra-model-based modified solutions.

Most of these models are variations of the same 2-D-DPD model, which is based on the frequency-selective assumption that the two bands of interest, their intermodulation, and harmonics by-products lie on separate frequencies. This assumption had been valid based on the practical bandwidth of most of the designed high-power PAs, which provides bands up to $100-500 \mathrm{MHz}$ apart while communication frequencies are in the gigahertz range. Fig. 1(a) shows the output of a nonlinear transmitter in dual-band concurrent operation in a commonly assumed scenario where the two bands lie on uncorrelated frequencies. Due to the interaction of signals at two bands, intermodulation and cross-modulation products are generated. The terms that fall outside the band-of-interest can be easily eliminated using a filter. Similarly, due to the imposed assumption of separate frequencies, the harmonics generated due to the signals also seem to fall outside the band of interest, and therefore, researchers sought to remove the distortions due to only the PA nonlinearities, which affect the in-band signals via distortion and cross-modulation [14]-[20].

However, recently, research in PA design is pushing towards ultra-wideband application [26], which, in turn, raised concern of interference from harmonics, which might fall within the working range of ultra-wideband power. Keeping this futuristic trend in mind, there had been very few recent attempts to model PA harmonics [27]. Harmonic cancellation feedforward techniques are proposed in [28] by injecting an uncorrelated signal at harmonic frequency, whereas [29] proposed a tunable third harmonic elimination technique for envelope-tracking amplifiers. However, to the best of the authors' knowledge, the interference of harmonics has not been studied in dual-band concurrent transmitters. Therefore, this paper proposes a 2D-HMP model for modeling and DPD for the practical scenario shown in Fig. 1(b) when one of the harmonics of the signal interferes with the upper band and cannot be removed using RF filters as proposed in concurrent DPD literature.

This paper is organized as follows. Section II describes the harmonic 2-D memory polynomial (2D-MP) model. Section III explains the measurement setup and demonstrates

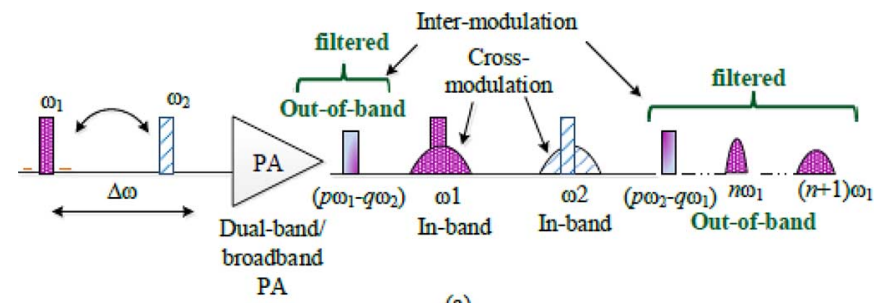

(a)

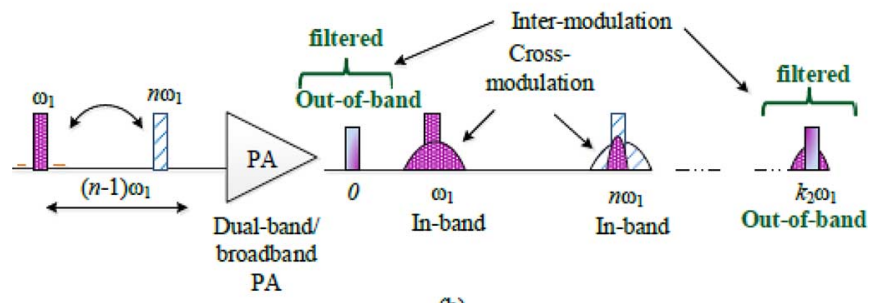

(b)

Fig. 1. (a) Commonly studied scenario, where two frequencies of dual-band operation are uncorrelated. (b) Scenario studied in this paper, where the harmonic of one signal interferes with the second band signal.

the effect of an harmonic interference for the example case of a second harmonic. Section IV reports the modeling performance of proposed models as compared to 2-D memory polynomial (2D-MP) model. Section V reports on the measurement results and their analysis. Section VI concludes this paper with a summary of the results achieved.

\section{Dual-Band Model With HaRmonic Frequencies}

\section{A. State-of-the-Art Two-Band Fundamental Envelope PA Model}

Before developing a two-band model for a PA, it is useful to first consider the case where a two-tone input excitation $a_{\text {in }}\left(\omega_{1}\right)$ and $a_{\text {in }}\left(\omega_{2}\right)$ at frequency $\omega_{1}$ and $\omega_{2}$, respectively, is applied at the input port of the PA.

It can be verified that the resulting odd-order intermodulation terms of the output $b_{\text {out }}$ below $\omega_{1}$ can be described with a Volterra system [12] as

$$
\begin{aligned}
b_{\text {out }}\left(\frac{p+1}{2} \omega_{1}-\frac{p-1}{2} \omega_{2}\right) & \\
= & V_{p}^{-} \cdot a_{\mathrm{in}}^{(p+1) / 2}\left(\omega_{1}\right) \cdot a_{\mathrm{in}}^{*(p-1) / 2}\left(\omega_{2}\right)
\end{aligned}
$$

with $p$ being an odd integer.

Explicit equations for the above terms up to seventh order are given by

$$
\begin{aligned}
b_{\text {out }}\left(4 \omega_{1}-3 \omega_{2}\right) & =V_{7}^{-} \cdot a_{\text {in }}^{4}\left(\omega_{1}\right) \cdot a_{\text {in }}^{* 3}\left(\omega_{2}\right) \\
b_{\text {out }}\left(3 \omega_{1}-2 \omega_{2}\right) & =V_{5}^{-} \cdot a_{\text {in }}^{3}\left(\omega_{\text {in }}\right) \cdot a_{\text {in }}^{* 2}\left(\omega_{2}\right) \\
b_{\text {out }}\left(2 \omega_{1}-\omega_{2}\right) & =V_{3}^{-} \cdot a_{\text {in }}^{2}\left(\omega_{1}\right) \cdot a_{\text {in }}^{*}\left(\omega_{2}\right) \\
b_{\text {out }}\left(\omega_{1}\right) & =V_{1}^{-} \cdot a_{\text {in }}\left(\omega_{1}\right) .
\end{aligned}
$$

Similarly the resulting odd-order intermodulation terms $b_{\text {out }}$ above $\omega_{2}$ at the PA output can be described with a Volterra system [12] as

$$
\begin{aligned}
& b_{\text {out }}\left(\frac{p+1}{2} \omega_{2}-\frac{p-1}{2} \omega_{1}\right) \\
& =V_{p}^{+} \cdot a_{\mathrm{in}}^{(p+1) / 2}\left(\omega_{2}\right) \cdot a_{\mathrm{in}}^{*(p-1) / 2}\left(\omega_{1}\right)
\end{aligned}
$$


with the explicit expansion of the terms up to seventh order given by

$$
\begin{aligned}
b_{\text {out }}\left(\omega_{2}\right) & =V_{1}^{+} \cdot a_{\text {in }}\left(\omega_{2}\right) \\
b_{\text {out }}\left(2 \omega_{2}-\omega_{1}\right) & =V_{3}^{+} \cdot a_{\text {in }}^{2}\left(\omega_{2}\right) \cdot a_{\text {in }}^{*}\left(\omega_{1}\right) \\
b_{\text {out }}\left(3 \omega_{2}-2 \omega_{1}\right) & =V_{5}^{+} \cdot a_{\text {in }}^{3}\left(\omega_{2}\right) \cdot a_{\text {in }}^{* 2}\left(\omega_{1}\right) \\
b_{\text {out }}\left(4 \omega_{2}-3 \omega_{1}\right) & =V_{7}^{+} \cdot a_{\text {in }}^{4}\left(\omega_{2}\right) \cdot a_{\text {in }}^{* 3}\left(\omega_{1}\right)
\end{aligned}
$$

where the Volterra functions $V_{k}^{ \pm}=V_{k}^{ \pm}\left(\left|a_{\text {in }}\left(\omega_{1}\right)\right|^{2},\left|a_{\text {in }}\left(\omega_{2}\right)\right|^{2}\right)$ are a function of the two envelopes. It can be noted from (1)-(10) that the various output components, which generally take the form $a_{\text {in }}\left(\omega_{1}\right)^{p} \cdot a_{\text {in }} *\left(\omega_{2}\right)^{q} \cdot\left|a_{\text {in }}\left(\omega_{1}\right)\right|^{k} \cdot\left|a_{\text {in }}\left(\omega_{2}\right)\right|^{m}$, lie on the same frequency location as $a_{\text {in }}\left(\omega_{1}\right)^{p} \cdot a_{\text {in }} *\left(\omega_{2}\right)^{q}$, and therefore a Volterra function comprised of absolute terms models the higher order terms at each intermodulation frequency location. This two-tone analysis assumes that the separation $\delta=\omega_{2}-\omega_{1}$ between the two tones verifies $\delta$ $\ll \omega_{1}$. This allows for the capture and mitigation of each of the inband and interband [intermodulation distortion (IMD)] signal components using a single receiver. It is also worth noticing that due to the small $\delta$, only odd-order intermodulations terms (third-order intermodulation distortion (IMD3), fifth-order intermodulation distortion (IMD5), ...) are the near-band terms of interest, while even-order terms (second-order intermodulation distortion (IMD2), fourth-order intermodulation distortion (IMD4), $\ldots)$ such as $\left((p+1) /(2) \omega_{2}+(p-1) /(2) \omega_{1}\right)$ are far enough in the frequency domain to be easily filtered out.

Subsequent dual-band literature based on the above theory [13]-[25] included a second assumption that the frequency separation $\delta$ was sufficiently large to allow for the filtering of all intermodulation terms. Therefore, most of the DPD efforts deals with the in-band distortion for the two-band case. However, this paper investigates the dual-band concurrent scenario shown in Fig. 1(b), where the two bands are separated by frequency separation equal or nearly equal to the harmonic of the first-band center frequency. In this scenario, none of the above assumptions hold, and therefore a new model is required for the accurate modeling of this concurrent ultra-wideband PA operation.

\section{B. Two-Tone Excitation With Harmonic Frequency Separation}

Consider the case of two modulated input signals $x_{1}$ and $x_{2}$ at the frequencies $\omega_{1}=\omega+\Delta$ and $\omega_{2}=2 \omega$ with $\Delta \ll \omega$. To identify the output intermodulation terms resulting near the two bands in concurrent operation, it is convenient to first consider the case of a narrowband two-tone signal at these center frequencies.

After performing a Volterra-series expansion for the near-band signal components, it is observed that both even and odd intermodulation components fall near the first and second bands. These terms can also be practically observed in Figs. 2 and 3, which show the intermodulation by-products generated near the bands of interest at the output of an ultra-wideband PA from Mini-Circuits (ZX60-14012L) when excited by a two-tone signal. The lower band single-tone signal $(\omega+\Delta) /(2 \pi)$ is at $978.4 \mathrm{MHz}+3.84 \mathrm{MHz}=982.24 \mathrm{MHz}$, while the upper band single-tone $2 \omega /(2 \pi)$ is as $1956.8 \mathrm{MHz}$. The corresponding terms in the frequency and time domains, along with their respective calculated amplitudes, are given in

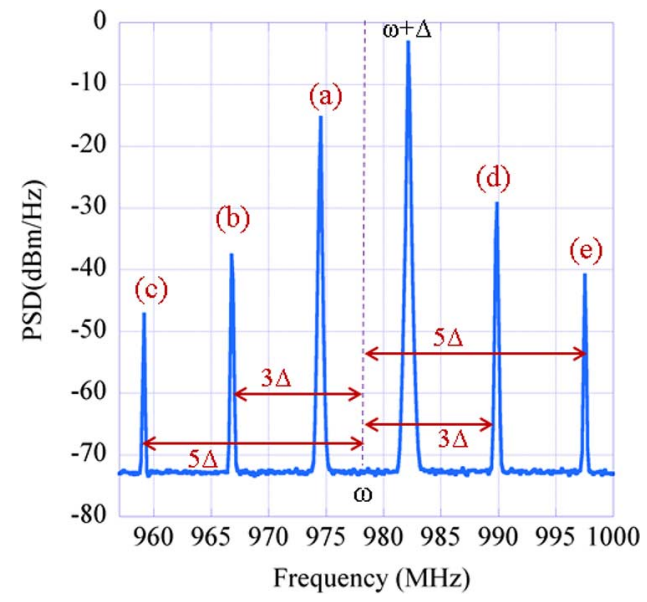

Fig. 2. Intermodulation terms interfering with the lower band $(\omega+\Delta)$.

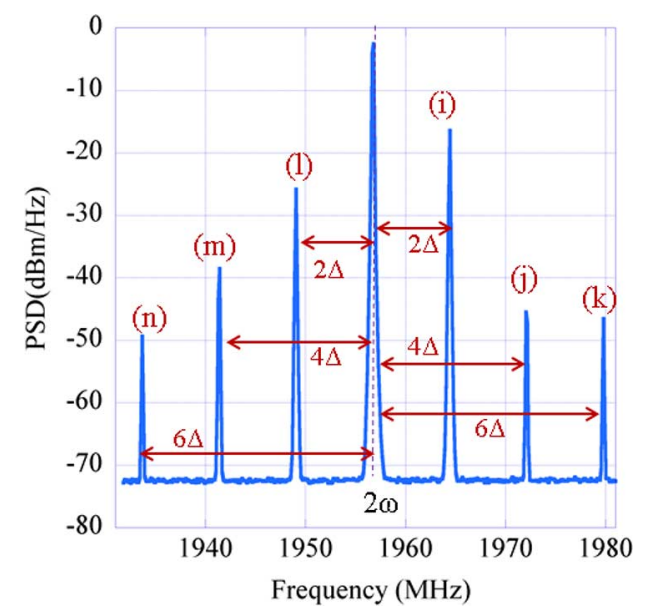

Fig. 3. Intermodulation terms interfering with the upper band $(2 \omega)$.

Table I where use is made of the notation $a_{\text {in }}(\omega)=x_{1} \exp (j \Delta t)$ and $a_{\text {in }}(2 \omega)=x_{2}$. From the intermodulation terms calculated at the output of the nonlinear system (as summarized in Table I), a trend can be observed for even and odd intermodulation terms. These terms can be summarized in four groups containing both even- and odd-order intermodulation terms. For $q=1,2, \ldots, n$, the lower band lower frequency side components [(a), (b), and (c) in Fig. 2] are given as

$$
b_{\text {out }}\left(\omega_{1}-2 q \Delta\right)=V_{12}^{(q)} \cdot x_{2}^{q} \cdot x_{1}^{*(2 q-1)} .
$$

The lower band upper frequency side components [(d) and (e) in Fig. 2] are given as

$$
b_{\text {out }}\left(\omega_{1}+2 q \Delta\right)=V_{13}^{(q)} \cdot x_{1}^{2 q+1} \cdot x_{2}^{* q} .
$$

The upper band lower frequency side components [(1), (m), and (n) in Fig. 3] are given as

$$
b_{\text {out }}\left(\omega_{2}-2 q \Delta\right)=V_{22}^{(q)} \cdot x_{2}^{q+1} \cdot x_{1}^{* 2 q} .
$$

The upper band, upper frequency side components [(i), (j), and (k) in Fig. 3] are given as

$$
b_{\text {out }}\left(\omega_{2}+2 q \Delta\right)=V_{23}^{(q)} \cdot x_{1}^{2 q} \cdot x_{2}^{*(q-1)} .
$$


TABLE I

INTERMODULATION TERMS INTERFERING WITH BANDS OF INTEREST

\begin{tabular}{|l|l|l|l|l|l|l|}
\hline Order & \multirow{2}{*}{ Lower Band } & \multicolumn{2}{c|}{ Upper Band } \\
& $\#$ & Frequency & IMD term & \# & Frequency & IMD term \\
\hline 2 & (a) & $\omega_{2}-\omega_{1}$ & $x_{2} x_{1}^{*} e^{j(\omega-\Delta) t}$ & (i) & $2 \omega_{1}$ & $x_{1}^{2} e^{j(2 \omega+2 \Delta) t}$ \\
5 & (b) & $2 \omega_{2}-3 \omega_{1}$ & $x_{2}^{2} x_{1}^{* 3} e^{j(\omega-3 \Delta) t}$ & (j) & $4 \omega_{1}-\omega_{2}$ & $x_{1}^{4} x_{2}^{*} e^{j(2 \omega+4 \Delta) t}$ \\
8 & (c) & $3 \omega_{2}-5 \omega_{1}$ & $x_{2}^{3} x_{1}^{* 5} e^{j(\omega-5 \Delta) t}$ & (k) & $6 \omega_{1}-2 \omega_{2}$ & $x_{1}^{6} x_{2}^{* 2} e^{j(2 \omega+6 \Delta) t}$ \\
4 & (d) & $3 \omega_{1}-\omega_{2}$ & $x_{1}^{3} x_{2}^{*} e^{j(\omega+3 \Delta) t}$ & (l) & $2 \omega_{2}-2 \omega_{1}$ & $x_{2}^{2} x_{1}^{* 2} e^{j(2 \omega-2 \Delta) t}$ \\
7 & (e) & $5 \omega_{1}-2 \omega_{2}$ & $x_{1}^{5} x_{2}^{* 2} e^{j(\omega+5 \Delta) t}$ & (m) & $3 \omega_{2}-4 \omega_{1}$ & $x_{2}^{3} x_{1}^{* 4} e^{j(2 \omega-4 \Delta) t}$ \\
\hline
\end{tabular}

Note that the Volterra functions $V_{b, p}^{(q)}$ are a function of the envelope of both bands

$$
V_{b, p}^{(q)}=V_{b, p}^{(q)}\left[\left|a_{\text {in }}(\omega)\right|^{2},\left|a_{\text {in }}(2 \omega)\right|^{2}\right] .
$$

The above IMD2 and IMD 3 components and harmonic of the lower band signals can be differentiated in the frequency domain in a two-tone test as these are narrowband signals. However, when a modulated signal with a wider bandwidth is used, then some of these nonlinear components superimpose with the signal of interest, and therefore the effect of these terms have to be included in the model to correctly represent the PA behavioral model when excited by two signals with harmonic frequency separation. It is also to be noted that for the $\Delta=0$ case, all the IMD terms completely superimpose with the signals of interest so that they cannot be filtered and need to be digitally corrected. The salient point of this work is that whenever two signals are used concurrently with near harmonic frequency separation, the IMD terms cannot be filtered out, and therefore the model should include the effective IMD and harmonic terms interfering with the signal. Note also that power back-off does not provide for an efficient distortion mitigation in the presence of harmonic interferences due to the dominance of the IMD2 terms. Indeed a $20-\mathrm{dB}$ reduction in IMD2 calls for a $20-\mathrm{dB}$ increase in the P1 dB of the PA unlike for IMD3 where a $10-\mathrm{dB}$ increase is sufficient.

This paper concentrates on a second harmonic interference case because the frequency range of current communication components mostly cover only the fundamental and second harmonic and this, therefore, is more practical for a demonstration study. However, using similar analysis, the interfering IMD terms for signals at $\omega$ and $p \omega$ center frequency bands can be calculated. For the case of a second harmonic interference with $\Delta=0$, the Volterra model for the system can be compactly represented using

$$
\begin{aligned}
b_{\text {out }}(\omega) & =V_{1,1} \cdot a_{\text {in }}(\omega)+V_{1,2} \cdot a_{\text {in }}^{*}(\omega) \cdot a_{\text {in }}(2 \omega) \\
b_{\text {out }}(2 \omega) & =V_{2,1} \cdot a_{\text {in }}(2 \omega)+V_{2,2} \cdot a_{\text {in }}^{2}(\omega)
\end{aligned}
$$

where each of the Volterra functions $V_{b, p}$ (see [10]) is a function of four baseband terms beside the operating point

$$
\begin{aligned}
V_{b, p}=V_{b, p}\left[\left|a_{\mathrm{in}}(\omega)\right|^{2},\right. & \left|a_{\mathrm{in}}(2 \omega)\right|^{2}, \\
& \left.a_{\mathrm{in}}^{2}(\omega) a_{\mathrm{in}}^{*}(2 \omega), a_{\mathrm{in}}^{* 2}(\omega) a_{\mathrm{in}}(2 \omega)\right] .
\end{aligned}
$$

Note that inserting (18) into (16) and (17) provides all higher power terms at $\omega$ and $2 \omega$ frequencies. This compact formulation is also applicable to the case $\Delta \neq 0$ by using $a_{\text {in }}(\omega)=$ $x_{1} \exp (j \Delta t)$ and $a_{\text {in }}(2 \omega)=x_{2}$. For example the terms (a)-(c) are obtained from the product of $a_{\mathrm{in}}(2 \omega) a_{\mathrm{in}}^{*}(\omega)$ times a power of $a_{\mathrm{in}}^{* 2}(\omega) a_{\mathrm{in}}(2 \omega)$. The terms (d) and (e) are obtained from the product of $a_{\text {in }}(\omega)$ times a power of $a_{\mathrm{in}}^{2}(\omega) a_{\mathrm{in}}^{*}(2 \omega)$. The terms (i)-(k) are obtained from the product of $a_{\text {in }}^{2}(\omega)$ times a power of $a_{\mathrm{in}}^{2}(\omega) a_{\mathrm{in}}^{*}(2 \omega)$. The terms (l) and (m) are obtained from the product of $a_{\mathrm{in}}(2 \omega)$ times a power of $a_{\mathrm{in}}^{* 2}(\omega) a_{\mathrm{in}}(2 \omega)$.

It should be noted that for a nonlinear system with no singularity, the functions $V_{i, j}$ must be analytic. Thus, this requires using an expansion in terms of both the baseband term $a_{\mathrm{in}}^{2}(\omega) a_{\mathrm{in}}^{*}(2 \omega)$ and its conjugate $a_{\mathrm{in}}^{* 2}(\omega) a_{\mathrm{in}}(2 \omega)$ like in the polyharmonic model (PHD, also referred as $X$-parameters) [30]. Alternatively, the real and imaginary parts of $a_{\mathrm{in}}^{2}(\omega) a_{\mathrm{in}}^{*}(2 \omega)$ could be used. Finally, another approach like in the Cardiff model [31] is to use the amplitude $\left|a_{\mathrm{in}}^{* 2}(\omega) a_{\mathrm{in}}(2 \omega)\right|$ and phase $\angle\left\{a_{\mathrm{in}}^{* 2}(\omega) a_{\mathrm{in}}(2 \omega)\right\}$. Given that we can rewrite the amplitude $\left|a_{\text {in }} * 2(\omega) a_{\text {in }}(2 \omega)\right|=\left|a_{\text {in }}(\omega)\right|^{2} \cdot\left|a_{\text {in }}(2 \omega)\right|$, it then becomes computationally desirable to replace the 4-D dependence of $V_{i, j}$ by the following 3-D dependence:

$$
V_{i, j}=V_{i, j}\left[\left|a_{\text {in }}(\omega)\right|,\left|a_{\text {in }}(2 \omega)\right|, \angle\left\{a_{\text {in }}^{2}(\omega) a_{\text {in }}^{*}(2 \omega)\right\}\right] .
$$

The use of $\left|a_{\text {in }}\right|$, which is nonanalytic (does not admit a power series expansion in terms of $a_{\text {in }}$ ), yields actually a more general formulation than the analytic Volterra model using $\left|a_{\text {in }}\right|^{2}$ since its includes it as a subset. It has been demonstrated that a nonanalytic formulation using $\left|a_{\text {in }}\right|$ is more suited to represent systems exhibiting discontinuities [32]. Note that quasi-slope discontinuities originate in a field-effect transistor (FET) PA from the voltage or current clipping when the FET saturates (drain current in the triode region) or in the substhreshold region (gate voltage below the threshold voltage).

\section{2-D Harmonic Memory Polynomial (2D-HMP) Model}

Considering $\left(x_{1}, x_{2}\right)$ and $\left(y_{1}, y_{2}\right)$, the two input and output baseband signals, respectively, at the two carrier frequencies, the generalized complex-baseband input/output relationship of the state-of-the-art 2D-MP model for concurrent dual band, with $K$ th nonlinearity order, is expressed as [14]

$$
\begin{aligned}
y_{b}(n)= & \sum_{m=0}^{M-1} x_{b}(n-m) \\
& \cdot \sum_{k=0}^{K-1} \sum_{j=0}^{k} c_{m, k, j}^{(b)} \cdot\left|x_{b}(n-m)\right|^{k-j} \cdot\left|x_{p}(n-m)\right|^{j}
\end{aligned}
$$

where $b, p \in\{1,2\}, b \neq p ; b$ and $p$ represents the first band $b$ of interest and the second band $p$, respectively, $c_{m, k, j}^{(b)}$ are the coefficients of the model and $M$ represents the memory depth. This model captures the in-band cross-modulation between the 
two-band signals. However, when the two carrier frequencies are $\omega$ and $2 \omega$, respectively, then due to the presence of the new four IMD terms of (11)-(14), shown in Section II-B, the 2D-MP model is not sufficient. Accounting for these terms, a new 2D-HMP model is given as follows for the lower and upper band signals:

$$
\begin{aligned}
y_{1}(n)= & \sum_{m=0}^{M_{l}} V_{1,1}^{m, 0}(n-m) \cdot x_{1}(n-m) \\
& +\sum_{m=0}^{M_{l}} \sum_{q=1}^{Q_{l}} V_{1,2}^{m, q}(n-m) \cdot x_{2}{ }^{q}(n-m) \\
& \cdot x_{1}{ }^{*(2 q-1)}(n-m) \\
& +\sum_{m=0}^{M_{u}} \sum_{q=1}^{Q_{u}} V_{1,3}^{m, q}(n-m) \cdot x_{1}{ }^{2 q+1}(n-m) \\
& \cdot x_{2}{ }^{* q}(n-m) \\
y_{2}(n)= & \sum_{m=0}^{M} V_{2,1}^{m, 0}(n-m) \cdot x_{2}(n-m) \\
& +\sum_{m=0}^{M_{l}} \sum_{q=1}^{Q_{l}} V_{2,2}^{m, q}(n-m) \\
& \cdot x_{2}{ }^{q+1}(n-m) \cdot x_{1}{ }^{* 2 q}(n-m) \\
& +\sum_{m=0}^{M_{u}} \sum_{q=1}^{Q_{u}} V_{2,3}^{m, q}(n-m) \\
& \cdot x_{1}{ }^{2 q}(n-m) \cdot x_{2}{ }^{*(q-1)}(n-m)
\end{aligned}
$$

where $V_{b, p}^{m, q}(n)$ are functions of the form

$$
V_{b, p}^{m, q}(n)=V_{b, p}^{m, q}\left(\left|x_{1}(n)\right|,\left|x_{2}(n)\right|\right) .
$$

When using a polynomial expansion, we have

$$
\begin{aligned}
& V_{b, p}^{m, q}\left(\left|x_{1}(n)\right|,\left|x_{1}(n)\right|\right)=\sum_{k=0}^{K_{l}} \sum_{j=0}^{k} a_{k, j}^{b, p, m, q} \\
& \cdot\left|x_{2}(n-m)\right|^{k-j} \cdot\left|x_{1}(n-m)\right|^{j} .
\end{aligned}
$$

It can be observed from (21), (22), and (24) that for a captured input-output measurement data set, the model is linear with respect to its coefficients.

In (21) and (22), $Q_{l}$ and $Q_{u}$ represent the intermodulation product orders. Similar to 2-D DPD [14], the coefficients $a_{k, j}^{b, p, m q}$ for the lower $(b=1)$ and upper $(b=2)$ bands can be calculated using the least square (LS) method. In this paper, the LS extraction has been implemented according to the singular value decomposition (SVD) method.

Alternately, following (16) and (17), a time-selective memory implementation of the 3-D model can be obtained using

$$
\begin{aligned}
y_{1}(n)= & \sum_{m}^{M_{l}-1} V_{1,1}^{m}(n-m) \cdot x_{1}(n-m) \\
& +V_{1,2}^{m}(n-m) \cdot x_{1}(n-m)^{*} \cdot x_{2}(n-m) \\
y_{2}(n)= & \sum_{m}^{M_{u}-1} V_{2,1}^{m}(n-m) \cdot x_{2}(n-m) \\
& +V_{2,2}^{m}(n-m) \cdot x_{1}^{2}(n-m)
\end{aligned}
$$

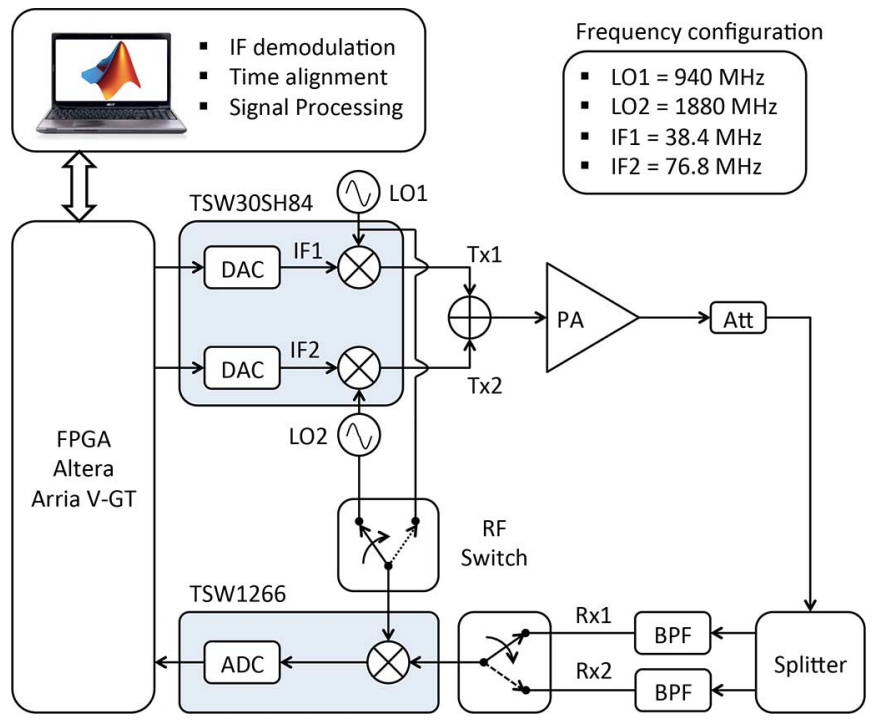

Fig. 4. Testbed setup to capture dual-band concurrent signal at harmonic distance.

where $V_{b, p}^{m}(n)$ are functions of the form

$$
V_{b, p}^{m}(n)=V_{b, p}^{m}\left(\left|x_{1}(n)\right|,\left|x_{2}(n)\right|, \theta_{12}(n)\right)
$$

with $\theta_{12}(n)=\angle\left\{x_{1}^{2}(n) x_{2}^{*}(n)\right\}$. With this 3-D implementation, the summation over $q$ in (21) and (22) is eliminated, but the dependence of the Volterra functions $V_{b, p}^{m}(n)$ over the phase $\theta_{12}(n)$ is added.

This alternative presentation can be implemented using polynomial or spline implementation. In this paper, results for alternative harmonic 3-D volterra (H3D-V) model is implemented using splines [15].

\section{Measurement Setup}

The measurement setup used for the concurrent dual-band operation is depicted in Fig. 4. A dual-channel transmitter (TSW30SH84) from Texas Instruments Incorporated is used for the concurrent dual-band transmission. The RF frequency range of each of the transmission channels is from $300 \mathrm{MHz}$ to $4 \mathrm{GHz}$. The dual-channel transmitter incorporates two 16-bit DACs, which share the same clock. The input data is stored in pre-allocated FPGA memories using MATLAB and Quartus software. The data from the Arria-V (GT series) FPGA used can be sent to TSW30SH84 board at the sampling rate of 307.2 MHz. The DAC in the transmit path is programmed with a sampling frequency of $1228.8 \mathrm{MHz}$, i.e., with an interpolation factor of 4 . The signal is upconverted using a TRF3705 quadrature modulator within the TSW30SH84 board and sent to an ultra-wideband PA. The output of the PA is downconverted and demodulated using a 500-MHz bandwidth TSW1266 receiver board from Texas Instruments Incorporated with a sampling rate of $614.4 \mathrm{MHz}$. The clock shared by the two DACs is also provided to the receiver ADCs.

A 10-MHz reference clock (Ref. clk) is used for the synchronization between the receiver and transmitter local oscillators (LOs). The signal is down-converted, digitized, and stored in the FPGA memory. The receiver board accepts an RF signal in the 


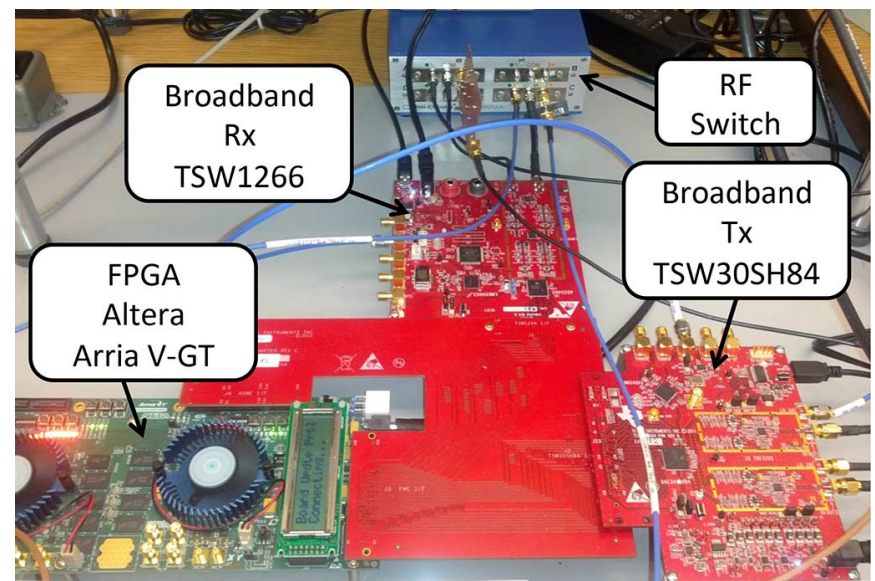

Fig. 5. Transceiver used for setup.

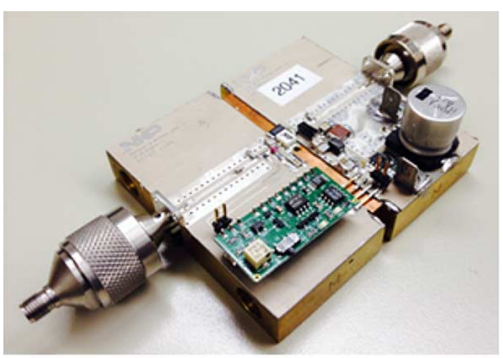

(a)

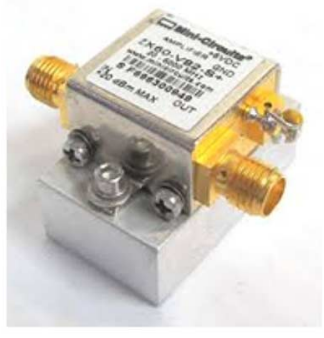

(b)
Fig. 6. DUTs. (a) 10-W peak-power PA from NXP. (b) ZX60-V82+ from Minicircuits.

frequency range of $1880-2390 \mathrm{MHz}$, therefore the second harmonic signal can be captured directly at the receiver. An input matching network was developed to extend the receiver operation in the fundamental band. An RF switch matrix from MiniCircuits is used before the receiver to select either the harmonic or fundamental frequency signal. The receiver of the feedback observation path captures one band at a time during the concurrent operation using a switch to select the proper $\mathrm{LO}$ and the RF input matching networks for the in-phase/quadrature (IQ) demodulator.

Fig. 5 shows a photograph of the testbed used for the measurement and Fig. 6 shows a photograph of the devices-under-test (DUTs).

\section{A. Devices and Signals Under Test}

Three different PAs are used for establishing the proof of concept, which are: 1) a ZX60-V82+ from Minicircuits with $20-6000-\mathrm{MHz}$ frequency operational range and $20-\mathrm{dBm}$ output at 1-dB gain compression; 2) a ZX60-14012L+ from Minicircuits with $0.4-14000-\mathrm{MHz}$ frequency operational range and $12.5-\mathrm{dBm}$ output at $1-\mathrm{dB}$ gain compression; and 3) a $10-\mathrm{W}$ peak-output-power PA from NXP with $500-2500-\mathrm{MHz}$ frequency operational range and $36-\mathrm{dBm}$ output at $1-\mathrm{dB}$ gain compression.

The PA is driven with a single-carrier WCDMA signal with a peak-to-average power ratio of $9.5 \mathrm{~dB}$ at $978.4 \mathrm{MHz}(940 \mathrm{MHz}$ $+38.4-\mathrm{MHz}$ IF shift) and 10-MHz long-term evolution (LTE) signal at $1956.8 \mathrm{MHz}(1880 \mathrm{MHz}+76.8 \mathrm{IF}$ shift $)$.

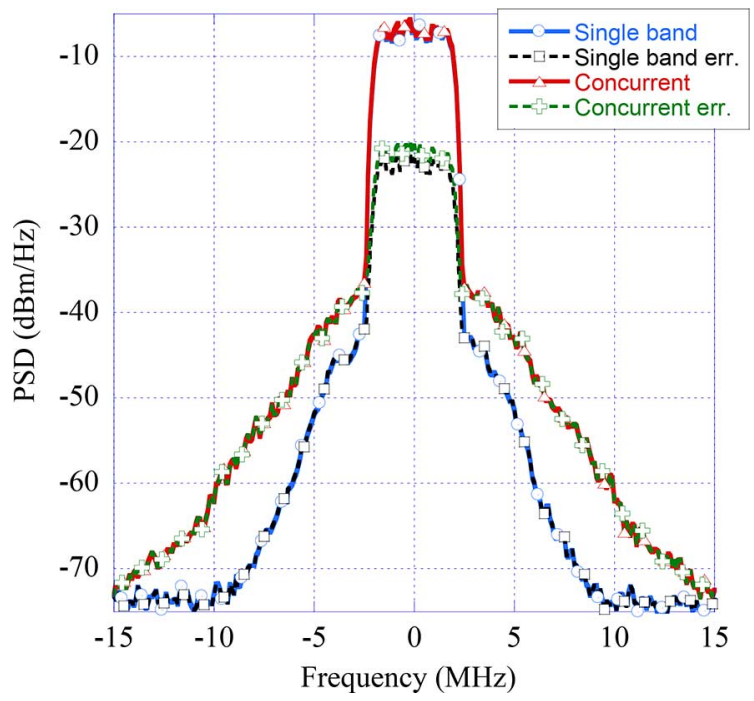

Fig. 7. Effect of concurrent operation at harmonic frequencies for lower band $(\omega)$.

\section{B. Time-Delay Alignment}

The time-delay alignment is done using a frequency-domain cross-correlation technique described in [27] and referenced therein.

The received signal contains nonlinear distortion and IMD terms. However, the PA is linear in the lower power ranges and the contribution from the signal power is dominant in the in-band signal component as compared to the distortions, therefore the linear correlation method is effective for the in-band data when both channels are active.

It is to be noted that the two signals used in concurrent operation are uncorrelated, and therefore the harmonic interference from the lower band signal is uncorrelated to the signal from the upper band. Moreover, the two signals are synchronized at the baseband level in the transmitter. Therefore, the cross-correlation method can be used individually in each band to calculate the time delay between the various transmitted and received signals. About the same group delays were observed between the transmitter and receiver in each channel. However, a relative group delay of four clocks was observed for the second harmonic propagating from the lower band to the upper band under some PA operation.

\section{EFfect of IMD Terms And Modeling Performance}

Figs. 7 and 8 show the effect of the interference of the IMD and cross-modulation terms for a single-carrier WCDMA signal in the lower band $(\omega)$ at $978.4 \mathrm{MHz}$ and a 10-MHz LTE signal at the upper band $(2 \omega)$ at $1916.8 \mathrm{MHz}$, respectively, while used in concurrent operation with the Zx60-14012-L+PA. The PA output for the upper band signal exhibits a gain compression of $0.6 \mathrm{~dB}$, while the lower band is compressed by $2.1 \mathrm{~dB}$. These figures show the power spectrum density (PSD) of the received output when each band is used individually (single-band operation, blue circle in online version) and when both bands are used concurrently (triangle, red in online version). In each case, the respective PSD of the transmission error is also plotted to observe the in-band and out-of-band effect of such terms; the 


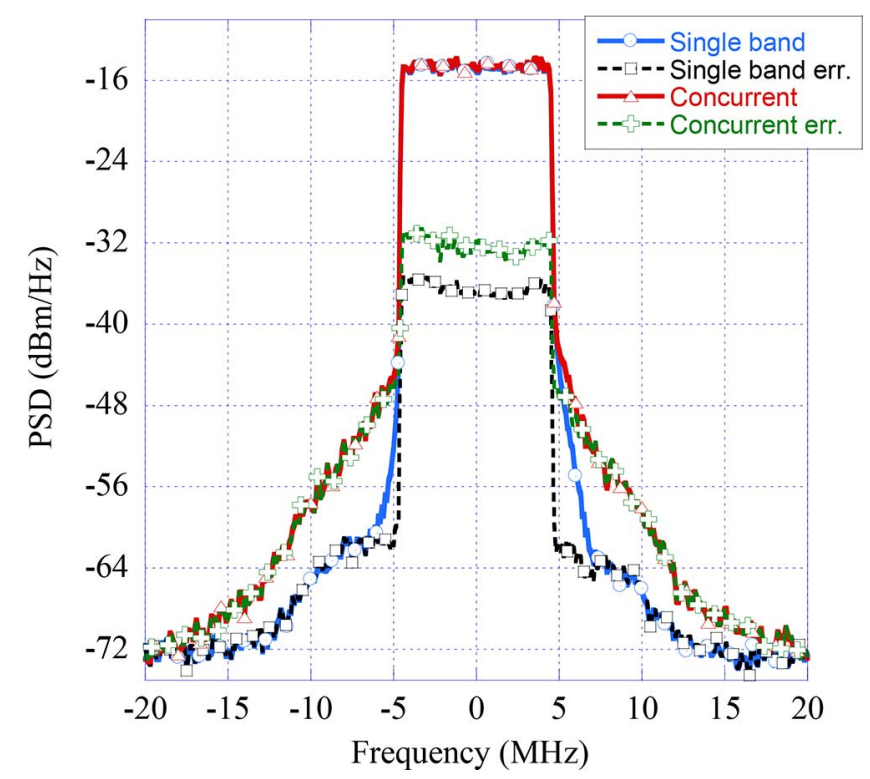

Fig. 8. Effect of concurrent operation at harmonic frequencies for upper band $(2 \omega)$.

transmission error is defined as the difference between the transmitted and received data.

Both the bands show a significant decrease in adjacent channel power ratio (ACPR) over the individual single-band transmission case. Moreover, the transmission error exhibits $2-5-\mathrm{dBc}$ performance degradation in-band, while 5-18-dBc performance degradation out-of-band due to the concurrent operation. From the two-tone analysis shown in Figs. 2 and 3 , it can be observed that for higher order IMD terms, the power of IMD components keep decreasing, and therefore it can be deduced that as $q$ in (11)-(14) for the two-tone system, and $Q_{u}$ and $Q_{l}$ in the modulated dual-band system given in (21) and (22) keep increasing, the corresponding IMD terms will have less impact on the received signal distortion. While Figs. 7 and 8 shows the case when two signals are at an exact harmonic separation, Figs. 9 and 10 show the output spectrum of the concurrent PA when the two frequencies are almost at an harmonic separation. The lower band frequency is at $(\omega /(2 \pi)+15.36 \mathrm{MHz})$, while the upper band frequency is at $2 \omega /(2 \pi)$. As in the two-tone analysis, the IMD terms can be identified. However, only the $(i)$ and $(l)$ terms for lower band frequency and only the $(a)$ term for the upper band frequency can be considered to make a significant contribution. The other terms, which are more than $50 \mathrm{dBc}$ below the main power, can be considered to have a minimal interference on the main signal. This observation helps in intuitively selecting the appropriate $Q_{u}$ and $Q_{l}$ ranges in the proposed model.

Table II shows the contribution of the different distortion terms (with $K_{u}, K_{l}=0$ ) in terms of normalized mean square error (NMSE) reduction for ZX60-14012L+ PA. Equations (21) and (22) show that the proposed model includes the cross-modulation terms of the 2D-MP model, as well as the first- and second-order IMD terms. Table II shows that in keeping with our observation, the first-order terms (1) in Fig. 2 and (a) in Fig. 3 provide the most significant NMSE improvement of 7-11 dB, however, as we keep going to higher IMD terms, the NMSE improvement decreases.

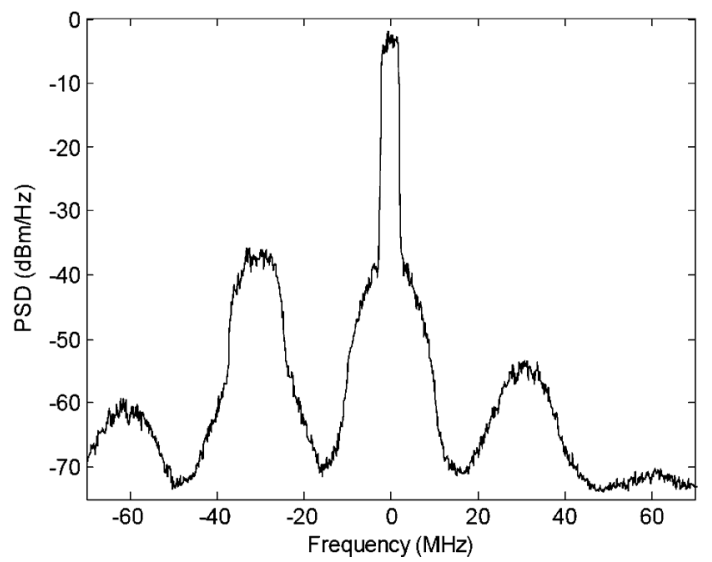

Fig. 9. Effect of concurrent operation at near harmonic frequencies for the lower band $(\omega+\Delta)$.

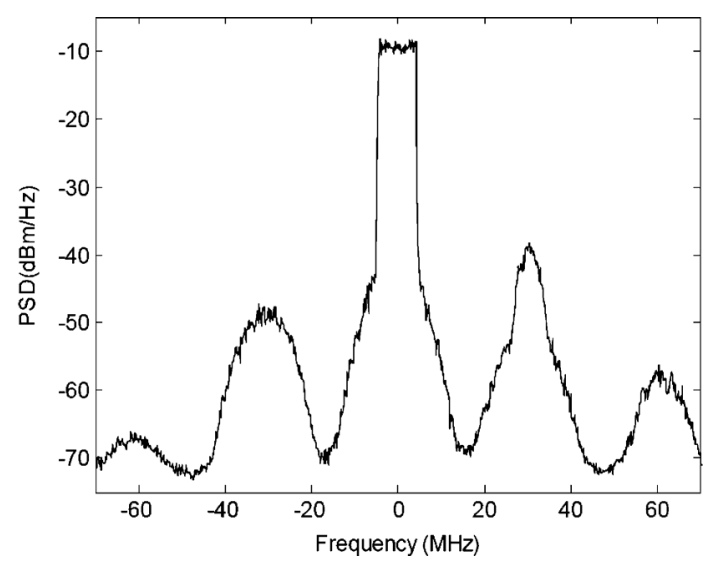

Fig. 10. Effect of concurrent operation at near harmonic frequencies for upper band $(2 \omega)$.

Table III shows the modeling performances of the 2D-MP model, the H2D-MP model, and its alternative presentation, the H3D-V model, for measured concurrent output data for different PAs and different nonlinearity conditions. The corresponding AM/AM (Gain) characteristics of these PA are shown in Figs. 11 and 12, respectively. In Table III, $K_{l}=K_{u}=K$ and $M_{l}=M_{u}=M$ for each model is denoted for the H2D-MP model using the notations given in Section II. Based on observation of Table II, $Q_{u}$ and $Q_{l}$ values are taken to be 1, except for the 10-W NXP PA when driven in saturation where a value of 2 is used. For the H3D-V model, $K_{n}$ represents the number of nodes for each dimension. Please note that, in both of the harmonic models, the number of coefficients can be further reduced by choosing different $K_{l}, K_{u}$, and $K_{n}$. It can be observed that the proposed H2D-MP model and its alternative representation provides almost similar performances, however, the number of coefficients needed vary for both the models according to the different PA characteristics. It is also noted that the H2D-MP model and H3D-V model provides 20-30-dB improvement in the NMSE as compared to the 2D-MP model. Increasing the number of coefficients for the 2D-MP model from 180 to 720 for $K=14$ only improves the NMSE by, at most, $1 \mathrm{~dB}$, indicating that this model has reached its performance saturation. On the other hand, the computer burden associated by the large number of coefficients required 
TABLE II

NMSE PeRformance (IN Decibels) OF DifFERENT Distortion TeRm INCLUSION IN H2D-MP

\begin{tabular}{|c|c|c|c|c|c|c|}
\hline \multirow[t]{2}{*}{ Dual-band concurrent models } & \multicolumn{3}{|c|}{$\begin{array}{c}\text { Lower Band } \\
\text { (WCDMA Signal at lower-band) }\end{array}$} & \multicolumn{3}{|c|}{$\begin{array}{c}\text { Upper Band } \\
\text { (LTE Signal at upper-band) }\end{array}$} \\
\hline & IMD term & PA & Inverse PA & IMD term & PA & Inverse PA \\
\hline 2DMP & Cross-modulation term & -28.9 & -27.5 & cross-modulation term & -28.1 & -26.9 \\
\hline+ Ist order terms & $\begin{array}{l}\text { (a) } x_{2} \cdot x_{1}^{*} f\left(\left|x_{1}\right|,\left|x_{2}\right|\right. \\
\text { (d) } x_{1}^{3} \cdot x_{2}^{*} f\left(\left|x_{1}\right|,\left|x_{2}\right|\right)\end{array}$ & $\begin{array}{l}-38.23 \\
-39.3\end{array}$ & $\begin{array}{l}-34.0 \\
-35.05\end{array}$ & $\begin{array}{l}\text { (i) } x_{1}^{2} f\left(\left|x_{1}\right|,\left|x_{2}\right|\right) \\
\text { (1) } x_{1}^{* 2} x_{2}^{2} f\left(\left|x_{1}\right|,\left|x_{2}\right|\right)\end{array}$ & $\begin{array}{l}-34.39 \\
-35.39\end{array}$ & $\begin{array}{l}-33.08 \\
-34.07\end{array}$ \\
\hline+ IInd order terms & $\begin{array}{l}\text { (b) } x_{2}^{* 2} \cdot x_{1}^{3} f\left(\left|x_{1}\right|,\left|x_{2}\right|\right) \\
\text { (c) } x_{2}^{* 2} \cdot x_{1}^{5} f\left(\left|x_{1}\right|,\left|x_{2}\right|\right)\end{array}$ & $\begin{array}{l}-39.39 \\
-39.45\end{array}$ & $\begin{array}{l}-35.07 \\
-35.22\end{array}$ & $\begin{array}{l}\text { (m) } x_{2}^{3} \cdot x_{1}^{* 4} f\left(\left|x_{1}\right|,\left|x_{2}\right|\right) \\
\text { (j) } x_{2}^{*} \cdot x_{1}^{4} f\left(\left|x_{1}\right|,\left|x_{2}\right|\right)\end{array}$ & $\begin{array}{l}-35.61 \\
-35.62\end{array}$ & $\begin{array}{l}-34.08 \\
-34.08\end{array}$ \\
\hline
\end{tabular}

TABLE III

NMSE Performance (in Decibels) Along With the Number of Coefficients (In Parentheses) Used for Proposed Harmonic Models, AS COMPARED TO THE 2D-DPD MODEL

\begin{tabular}{|c|c|c|c|c|c|c|}
\hline \multirow[t]{2}{*}{ UWB PAs } & \multicolumn{3}{|c|}{$\begin{array}{c}\text { Lower Band } \\
\text { (WCDMA Signal at lower-band) }\end{array}$} & \multicolumn{3}{|c|}{$\begin{array}{c}\text { Upper Band } \\
\text { (LTE Signal at upper-band) }\end{array}$} \\
\hline & 2D-MP & H2D-MP & H3D-V & 2D-MP & H2D-MP & H3D-V \\
\hline (1) NXP Nonlinear & $\begin{array}{l}-10.18 \\
K=7, M=4 \\
(180)\end{array}$ & $\begin{array}{l}-41.66 \\
K=7, M=4 \\
Q=2,(920)\end{array}$ & $\begin{array}{l}-40.56 \\
K_{n}=7, M=4 \\
(750)\end{array}$ & $\begin{array}{l}-12.08 \\
K=7, M=4 \\
(180)\end{array}$ & $\begin{array}{l}-44.09 \\
K=7, M=4 \\
Q=2,(920)\end{array}$ & $\begin{array}{l}-43.09 \\
K_{n}=7, M=4 \\
(750)\end{array}$ \\
\hline (2) NXP Mildly Nonlinear & $\begin{array}{l}-12.10 \\
K=7, M=4 \\
(180)\end{array}$ & $\begin{array}{l}-39.96 \\
K=7, M=4 \\
Q=1,(540)\end{array}$ & $\begin{array}{l}-37.24 \\
K_{n}=5, M=4 \\
(384)\end{array}$ & $\begin{array}{l}-21.49 \\
K=7, M=4 \\
(180)\end{array}$ & $\begin{array}{l}-43.51 \\
K=7, M=4 \\
Q=1,(540)\end{array}$ & $\begin{array}{l}-41.53 \\
K_{n}=5, M=4 \\
(384)\end{array}$ \\
\hline (3) ZX60-14012L+ & $\begin{array}{l}-26.40 \\
K=7, M=4 \\
(180)\end{array}$ & $\begin{array}{l}-47.9 \\
K=7, M=4 \\
Q=1,(540)\end{array}$ & $\begin{array}{l}-47.56 \\
K_{n}=7, M=4 \\
(750)\end{array}$ & $\begin{array}{l}-29.38 \\
K=7, M=4 \\
(180)\end{array}$ & $\begin{array}{l}-49.04 \\
K=7, M=4 \\
Q=1,(540)\end{array}$ & $\begin{array}{l}-49.44 \\
K_{n}=7, M=4 \\
(750)\end{array}$ \\
\hline
\end{tabular}
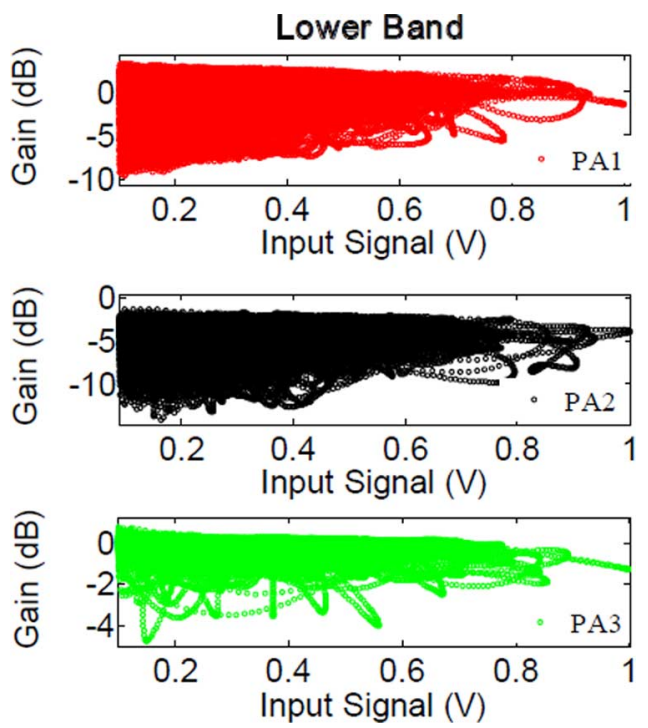

Fig. 11. Gain compression and distortion profile for three PAs used in Table III at lower band.

by the H2D-MP and H3D-V models is alleviated by the use of the SVD matrix inversion approach, which retains only the dominant SVD singular values.

Figs. 13 and 14 shows the PSD of the proposed H2D-MP model error compared to the PSD of the 2D-MP model error [14].

\section{MeAsurement Results FOR DPD APPLiCATION}

The DPD for the proposed model is implemented according to the indirect learning architecture (ILA). Fig. 15 shows the
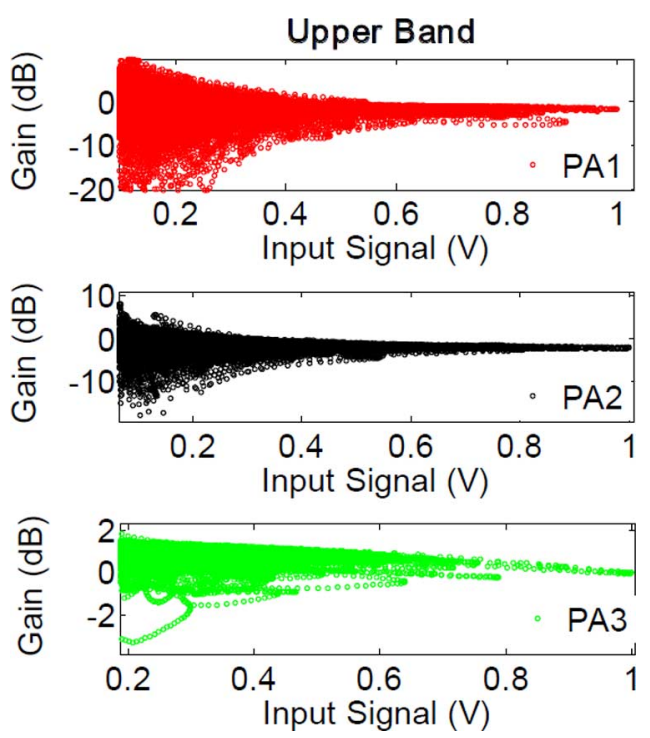

Fig. 12. Gain compression and distortion profile for three PAs used in Table III at upper band.

ILA for DPD. The input signal is passed through the predistorter $K$ with parameters $\alpha$, creating the predistorted signal $u(n)$. This predistorted signal $u(n)$ is passed through the PA to provide output $y(n)$. Output of the PA is provided as input to the predistorter $K$ to create $u \tilde{(n)}$. The required condition for linear PA operation is $y(n)=G x(n)$. The ILA works on the principle that, when $y(n) / G=x(n)$, then $\tilde{u(n)}=u(n)$.

For the first iteration, $u(n)=x(n)$, and DPD is a model that maps $x(n)=K(y(n) / G)$, where $K$ represents the predistorter nonlinear function. The modeling of the complex input, with 


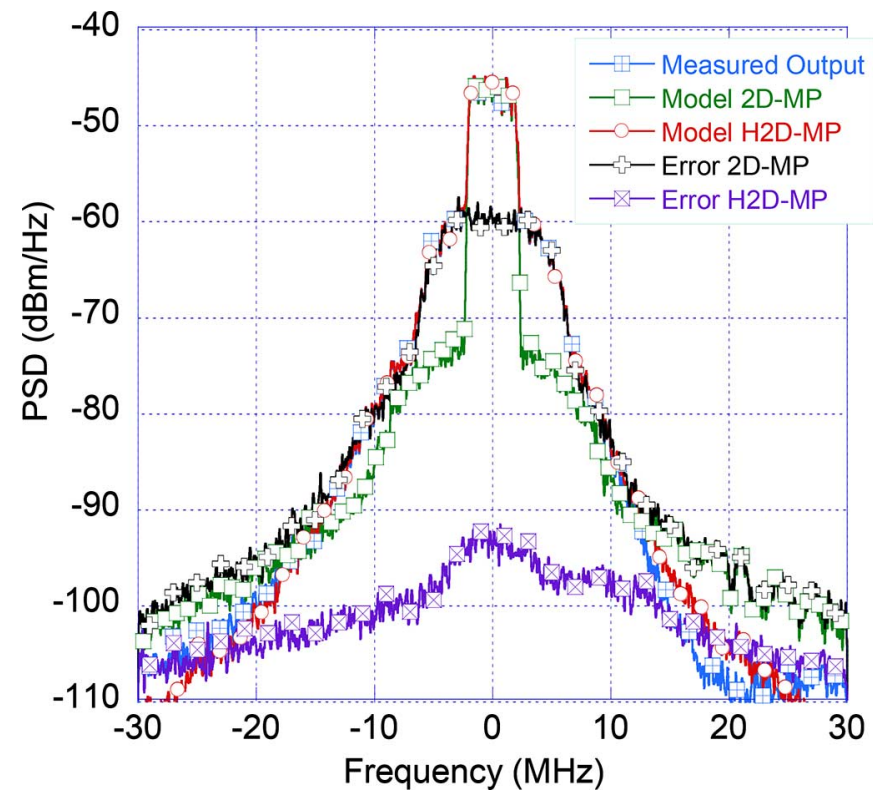

Fig. 13. Comparison of 2D-MP and H2D-MP model errors for lower band.

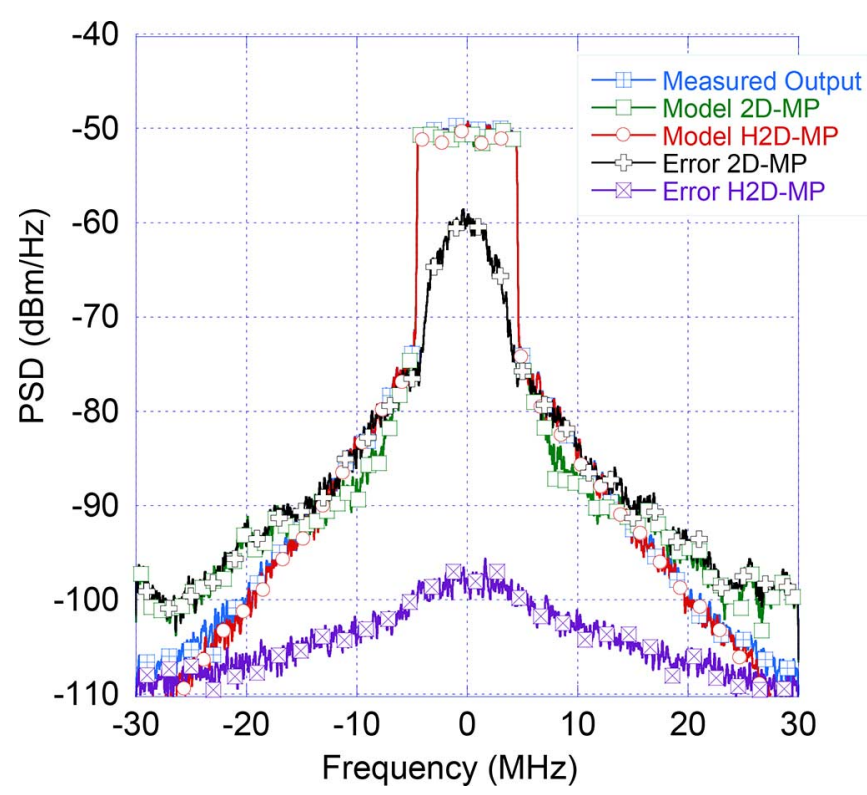

Fig. 14. Comparison of 2D-MP and H2D-MP model errors for upper band.

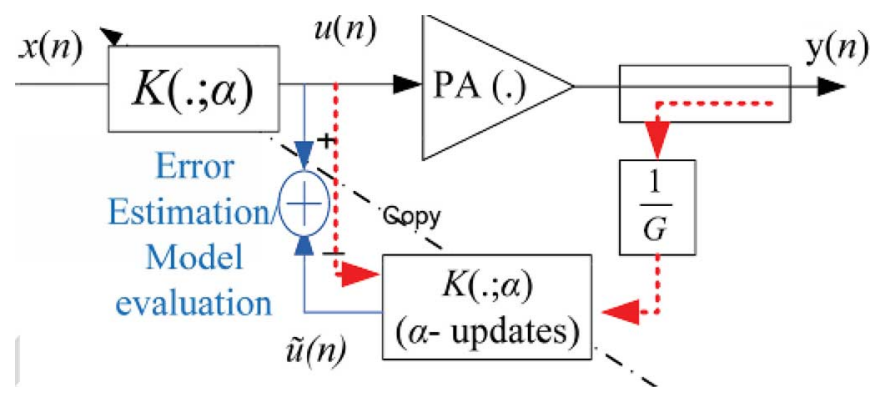

Fig. 15. Indirect learning architecture.

respect to the normalized PA output, is the inverse of the PA behavioral model; therefore, DPD modeling is also known as inverse modeling. This inverse modeling is the foremost require-
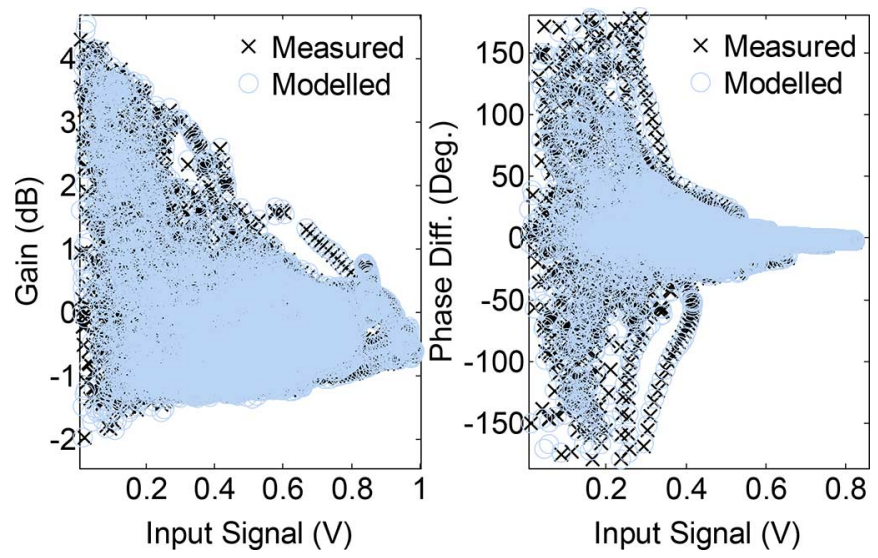

Fig. 16. PA inverse modeling performance with proposed 2D-HMP model shown for NXP PA for lower band.
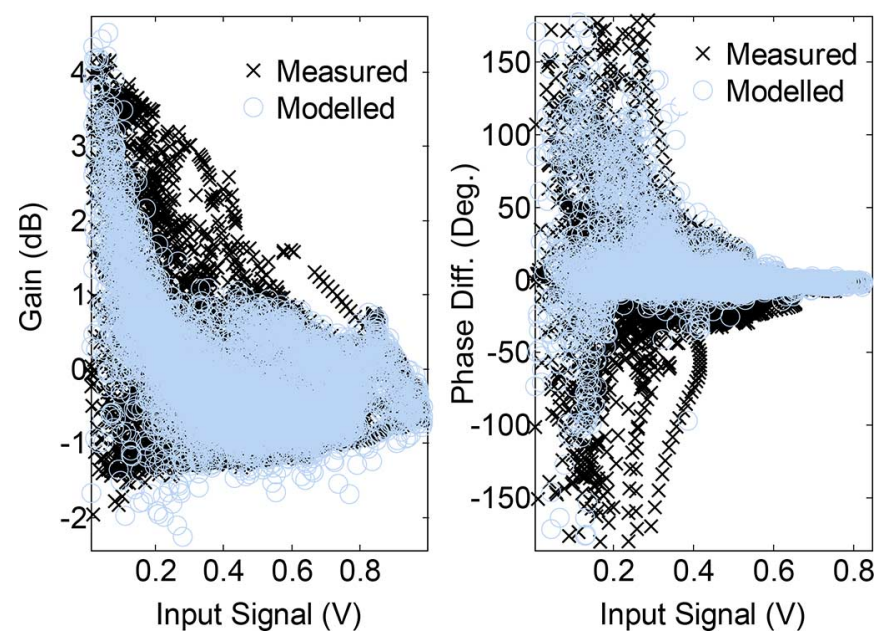

Fig. 17. PA inverse modeling performance with 2D-MP model shown for NXP PA for lower band.

ment for the successful application of a DPD model. The ILA architecture is applied to both the bands individually [17]-[19], [27] to achieve linearized output at both the bands.

Figs. 16 and 17 shows the PA inverse characteristics (AM/AM and $\mathrm{AM} / \mathrm{PM}$ ) modeled at the upper band for the proposed H2D-MP model, as well as the 2D-MP model in the presence of the second harmonic. It is to be noted that according to the ILA for DPD, any model providing a more accurate inverse model should be able to provide better DPD. It is evident that the 2D-MP model does not provide as good a performance as the proposed model due to the absence of harmonic and intermodulation terms and that it is anticipated that the H2D-MP model should provide better linearization results for concurrent transmission at frequency spacing corresponding to an harmonic tone. The proposed model is applied now for DPD application and the signal quality in the transmission is judged using two main figures of merits, ACPR, and NMSE. The ACPR used is the mean of the ACPR for the lower and upper channels, which is calculated as follows:

$$
\mathrm{ACPR}=\frac{\int_{\omega_{L}-\frac{\mathrm{BW}}{2}}^{\omega_{L}+\frac{\mathrm{BW}}{2}} Y^{2}(\omega) d \omega+\int_{\omega_{U}-\frac{\mathrm{BW}}{2}}^{\omega_{U}+\frac{\mathrm{BW}}{2}} Y^{2}(\omega) d \omega}{\int_{\omega-\frac{\mathrm{BW}}{2}}^{\omega+\frac{\mathrm{BW}}{2}} Y^{2}(\omega) d \omega}
$$




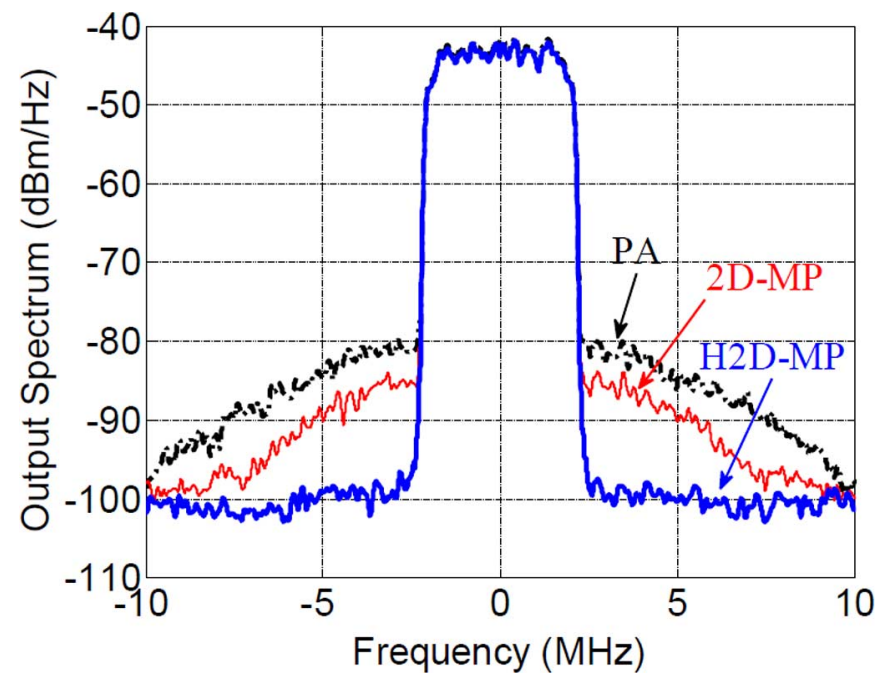

Fig. 18. DPD performance in terms of ACPR with the proposed H2D-MP model for lower band.

TABLE IV

Transmission ERror RedUCtion PERFormance

\begin{tabular}{|c|c|c|c|}
\hline & w/o DPD & 2DMP DPD & H2DMP DPD \\
\hline \hline LB(NMSE (dB)) & -22.6 & -33.1 & -44.3 \\
\hline UB(NMS (dB)) & -20.6 & -33.9 & -44.7 \\
\hline \hline LB (ACPR (dB)) & -35.2 & -39.1 & -53.7 \\
\hline UB(ACPR (dB)) & -33.8 & -41.7 & -51.5 \\
\hline
\end{tabular}

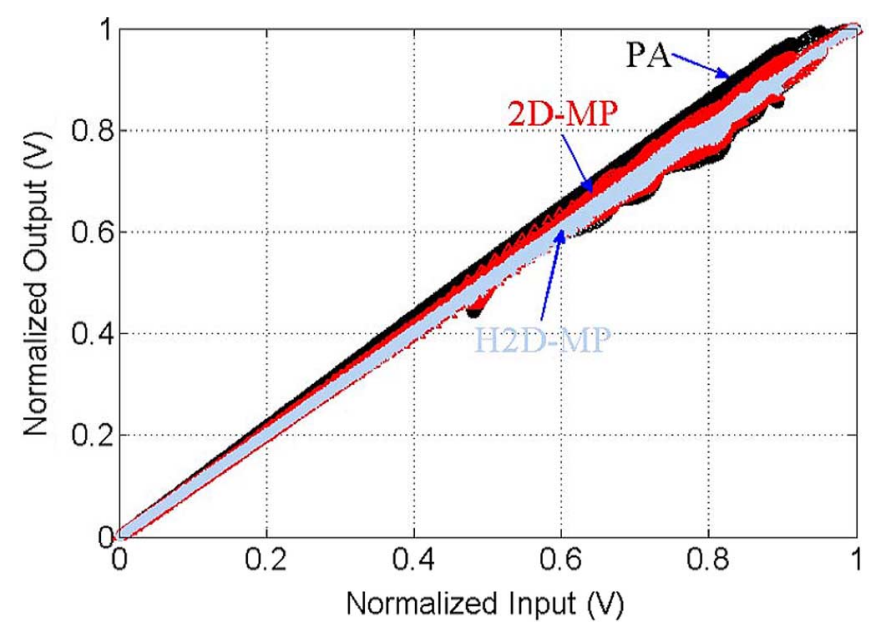

Fig. 19. DPD performance in terms of output power for H2D-MP model at lower band.

where $Y^{2}(\omega)$ represents the PSD of the signal, $\omega_{L}$ and $\omega_{U}$ represent, respectively, the center frequencies of lower and upper adjacent channel bands, and BW represents the radial bandwidth of the adjacent channel used for ACPR calculation. $\omega$ represents the center frequency of the signal. Figs. 18 and 22 show the out-of-band distortion cancellation for the lower and upper band for the Zx60-82+ PA using the 2D-MP and H2D-MP models with $K_{l}=K_{u}=k=6, Q_{l}=Q_{u}=1$, and $M=4$.

The second important figure-of-merit is the transmission error, which is defined by the error between the transmitted and received signals. For OFDM-based signals, the error vector magnitude (EVM) provides a metric to judge the in-band transmission error, however, for WCDMA-like signals, the

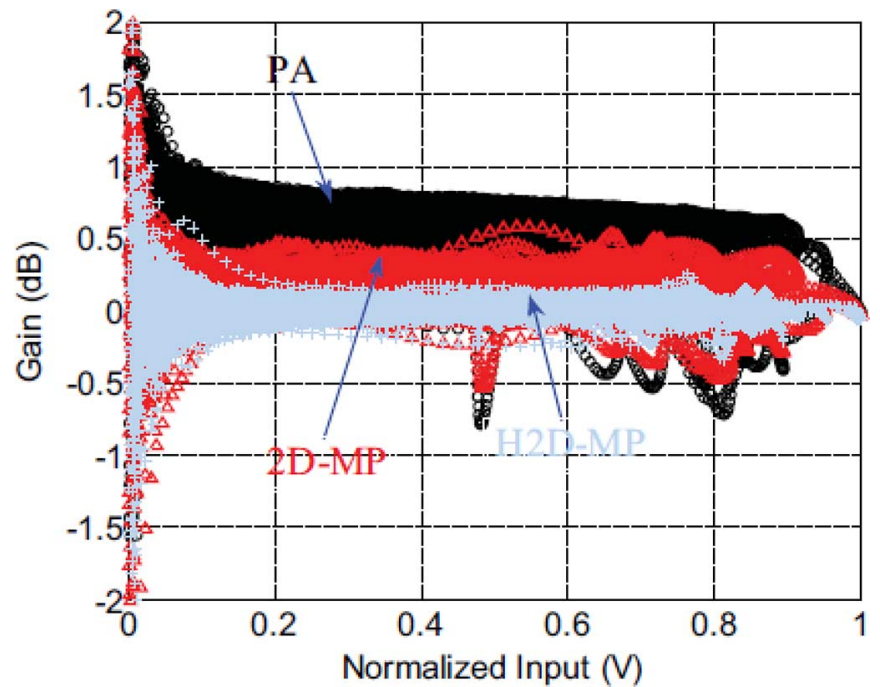

Fig. 20. DPD performance in terms of gain for H2D-MP model at lower band.

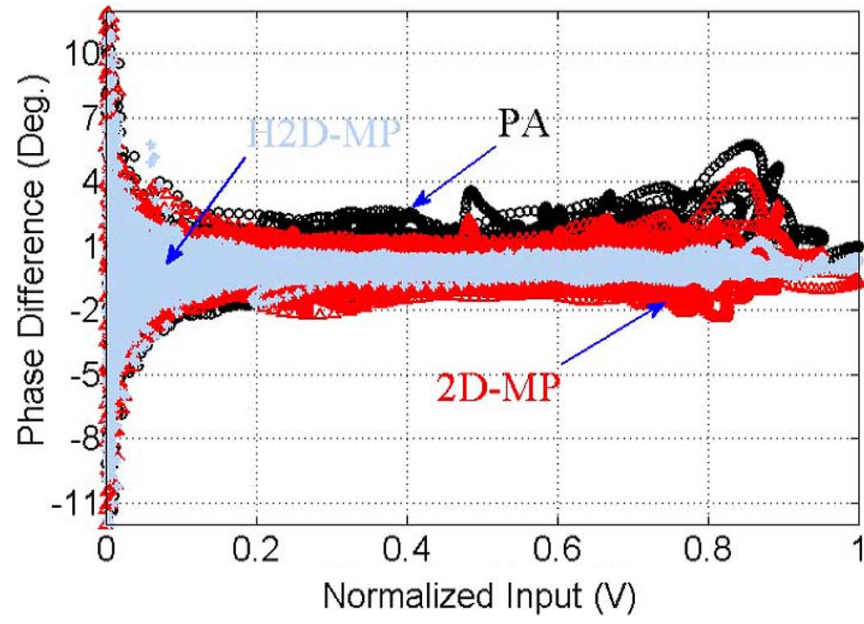

Fig. 21. DPD performance in terms of phase with the proposed 2D-HMP model at lower band.

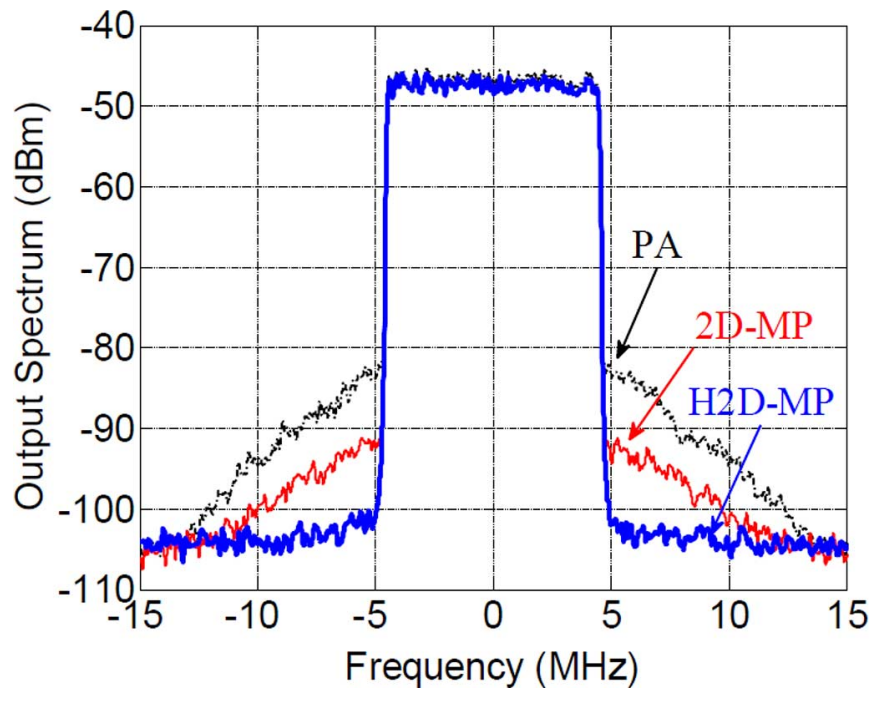

Fig. 22. DPD performance in terms of ACPR with the proposed 2D-HMP model for upper band.

constellation points are not defined and the NMSE is used as a metric to define the in-band transmission error [22]. 


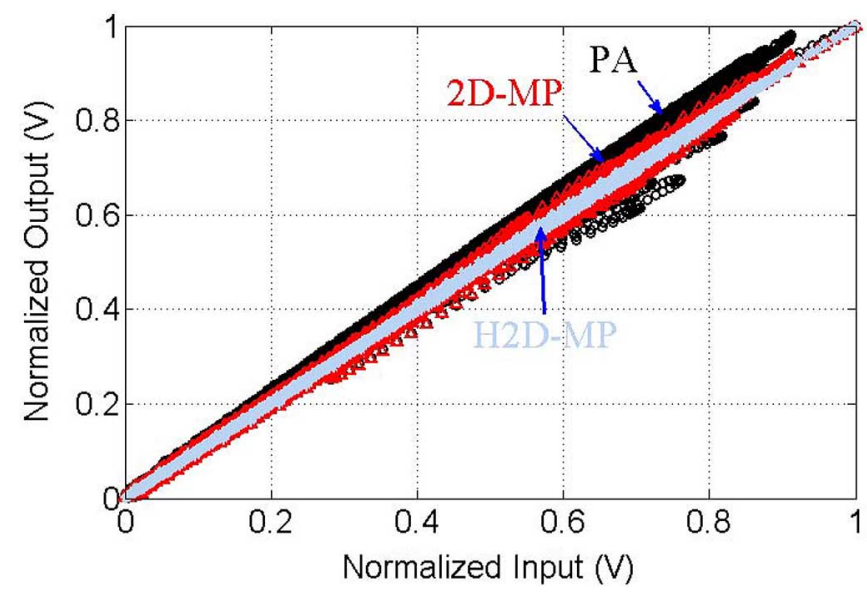

Fig. 23. DPD performance in terms of output power with the proposed 2D-HMP model for upper band.

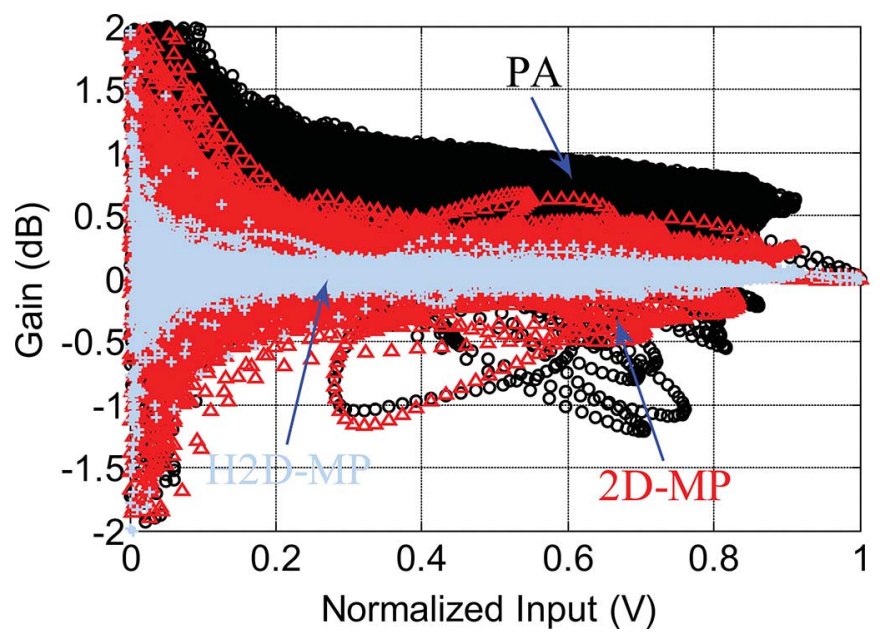

Fig. 24. DPD performance in terms of gain with the proposed 2D-HMP model for upper band.

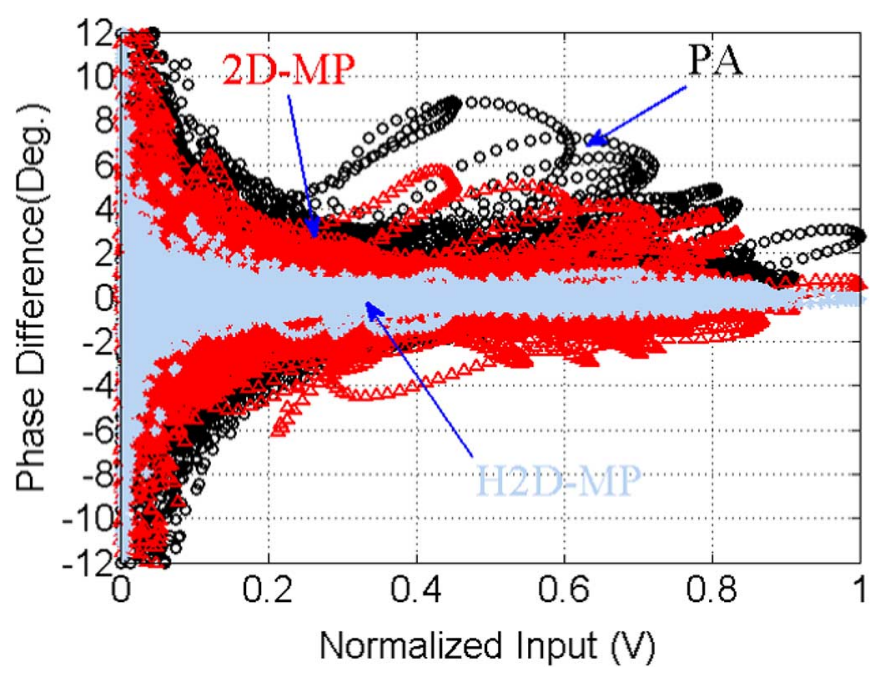

Fig. 25. DPD performance in terms of phase with the proposed 2D-HMP model for upper band.

Table IV shows the transmission error in terms of NMSE before and after DPD application to the two bands. For both bands, more than 22-dB improvement in terms of transmission
NMSE is observed. It is to be noted that the proposed model provides up to $11-\mathrm{dB}$ reduction in-band transmission error as compared to state-of-the-art 2D-MP-model-based DPD. Table IV also shows more than 10-dB improvement in the ACPR as compared to 2D-MP-model-based DPD. To visually observe the linearization results, Figs. 18-21 show the DPD performances in terms of output power, gain, and phase plots for the lower band signal. Likewise, Figs. 22-25 shows the DPD performances in terms of output power, gain, and phase plots for the upper band signal; a significant reduction in signal distortion can be observed.

It is to be noted that the H2D-MP model can be implemented in a FPGA according to the time-mutiplexing method given in [26], where additional IMD terms have to be included in memory space calculation apart from 2D-MP terms.

\section{CONCLUSION}

This paper has discussed the effect of concurrent dual-band transmission when the harmonic of the lower band signal directly interferes with the signal in the upper band such that it cannot be removed with an RF filter. As a proof-of-concept, the paper is focused on the case where the upper band signal is at the second harmonic of the lower band signal. This paper has proposed a 2D-MP model based on intermode analysis of a nonlinear PA for the dual-band concurrent transmission in the presence of harmonic interferences. Moreover, it is shown that similar IMD2 and IMD3 terms can also be achieved by a Volterra model synthesis and an alternative H3D-V model was proposed and implemented using splines. The effectiveness of the proposed models has been verified for different PAs and nonlinearity conditions. The proposed model has been utilized for the inverse modeling, and as a proof-of-concept, the DPD has been implemented using a low-cost FPGA-based system featuring a dual-channel transmitter and a single-feedback receiver. By taking the unavoidable intermodulation interference into account, the proposed model provides an NMSE improvement at the transmitter output of approximately $10-12 \mathrm{~dB}$ and an ACPR improvement up to $10-13 \mathrm{~dB}$ as compared to the state-of-the-art 2D-DPD model.

\section{ACKNOWLEDGMENT}

The first author would like to thank all the members of the Nonlinear RF Laboratory Group, The Ohio State University, for several technical discussions. The authors are grateful to Altera Inc., for the donation of the FPGA testbed used.

\section{REFERENCES}

[1] P. Roblin, C. Quindroit, N. Naraharisetti, S. Gheitanchi, and M. Fitton, "Concurrent linearization: The state of the art for modeling and linearization of multiband power amplifiers," IEEE Microw. Mag., vol. 14, no. 7, pp. 75-91, Nov. 2013.

[2] K. Rawat, M. S. Hashmi, and F. M. Ghannouchi, "Dual the band and optimize," IEEE Microw. Mag., vol. 13, no. 2, pp. 69-82, Apr. 2012.

[3] "Understanding the requirements for LTE-advanced carrier aggregation manufacturing test," Agilent Technol., Santa Clara, CA, USA, Appl. Note 5991-3762EN, Jan. 16, 2014.

[4] R. Liu, D. Schreurs, W. De Raedt, F. Vanaverbeke, and R. Mertens, "Concurrent dual-band power amplifier with different operation modes," in IEEE MTT-S Int. Microw. Symp. Dig., Baltimore, MD, USA, Jun. 2011, pp. 1-4. 
[5] K. Rawat and F. M. Ghannouchi, "Design methodology for dual-band Doherty power amplifier with performance enhancement using dualband offset lines," IEEE Trans. Ind. Electron., vol. 59, no. 12, pp. 4831-4842, Dec. 2012.

[6] D. Y. T. Wu and S. Boumaiza, "A modified Doherty configuration for broadband amplification using symmetrical devices," IEEE Trans. Microw. Theory Techn., vol. 60, no. 10, pp. 3201-3213, Oct. 2012.

[7] A. Cidronali, I. Magrini, R. Fagotti, and G. Manes, "A new approach for concurrent dual-band IF digital predistortion: System design and analysis," in Integr. Nonlinear Microw. Millim.-Wave Circuits Workshop, Nov. 24-25, 2008, pp. 127-130.

[8] P. Roblin, S. K. Myoung, D. Chaillot, Y. G. Kim, A. Fathimulla, J. Strahler, and S. Bibyk, "Frequency-selective predistortion linearization of RF power amplifiers," IEEE Trans. Microw. Theory Techn., vol. 56, no. 1, pp. 65-76, Jan. 2008.

[9] X. Yang, P. Roblin, D. Chaillot, S. Mutha, J. Strahler, J. Kim, and M. Ismail, "Fully orthogonal multi-carrier predistortion linearization for RF power amplifiers," in IEEE MTT-S Int. Microw. Symp. Dig., 2009, pp. 1077-1080.

[10] P. Roblin, Nonlinear RF Circuits and Nonlinear Vector Network Analyzers Interactive Measurement and Design Techniques. Cambridge, U.K.: Cambridge Univ. Press, 2011, pp. 621-625.

[11] J. Kim, P. Roblin, X. Yang, and D. Chaillot, "A new architecture for frequency-selective digital predistortion linearization for RF power amplifiers," in IEEE MTT-S Int. Microw. Symp. Dig., Montreal, QC, Canada, Jun. 2012, pp. 1-3.

[12] J. Kim, P. Roblin, D. Chaillot, and Z. Xie, "A generalized architecture for the frequency-selective digital predistortion linearization technique," IEEE Trans. Microw. Theory Techn., vol. 61, no. 1, pp. 596-605, Jan. 2013.

[13] S. A. Bassam, W. Chen, M. Helaoui, F. M. Ghannouchi, and Z. Feng, "Linearization of concurrent dual-band power amplifier based on 2D-DPD technique," IEEE Microw. Wireless Compon. Lett., vol. 21, no. 12 , pp. $685-687$, Dec. 2011.

[14] S. A. Bassam, M. Helaoui, and F. M. Ghannouchi, "2-D digital predistortion (2-D-DPD) architecture for concurrent dual-band transmitters," IEEE Trans. Microw. Theory Techn., vol. 59, no. 10, pp. 2547-2553, Oct. 2011

[15] N. Naraharisetti, C. Quindroit, P. Roblin, S. Gheitanchi, V. Mauer, and M. Fitton, "2-D digital predistortion (2-D-DPD) architecture for concurrent dual-band transmitters," in IEEE MTT-S Int. Microw. Symp. Dig., Jun. 2013, pp. 2547-2553.

[16] M. Rawat, N. Narharishetti, C. Quindroit, P. Roblin, R. Pond, K. Salam, and C. Xie, "Dual-band transmitter behavioral modeling with physically motivated 2-D rational functions," in 82nd ARFTG Microw. Meas. Symp., Columbus, OH, USA, Nov. 2013, pp. 1-4.

[17] A. K. Kwan, S. A. Bassam, M. Helaoui, and F. M. Ghannouchi, "Concurrent dual band digital predistortion using look up tables with variable depths," in Proc. IEEE Power Amplifiers for Wireless Radio Appl. Top. Conf., Santa Clara, CA, USA, Jan. 2013, pp. 25-27.

[18] S. A. Bassam, A. Kwan, W. Chen, M. Helaoui, and F. M. Ghannouchi, "Subsampling feedback loop applicable to concurrent dual-band linearization architecture," IEEE Trans. Microw. Theory Techn., vol. 60 no. 6, pp. 1990-1999, May 2012.

[19] Y. J. Liu, W. Chen, B. Zhou, J. Zhou, and F. M. Ghannouchi, "2D augmented Hammerstein model for concurrent dual-band power amplifiers," Electron. Lett., vol. 48, pp. 1214-1216, Oct. 2012.

[20] Y.-J. Liu, W. Chen, J. Zhou, B. H. Zhou, and F. Ghannouchi, "Digital predistortion for concurrent dual-band transmitters using 2-D modified memory polynomials," IEEE Trans. Microw. Theory Techn., vol. 61, no. 1, pp. 281-290, Jan. 2013.

[21] S. Zhang, W. Chen, F. M. Ghannouchi, and Y. Chen, "An iterative pruning of 2-D digital predistortion model based on normalized polynomial terms," in IEEE MTT-S Int. Microw. Symp. Dig., Jun. 2013, pp. $1-4$.

[22] C. Quindroit, N. Naraharisetti, P. Roblin, S. Gheitanchi, V. Mauer, and M. Fitton, "FPGA implementation of orthogonal 2D digital predistortion system for concurrent dual-band power amplifiers based on time-division multiplexing," IEEE Trans. Microw. Theory Techn., vol. 61, no. 12, pp. 4591-4599, Dec. 2013.

[23] M. Rawat, K. Rawat, M. Younes, and F. M. Ghannouchi, "Joint mitigation of nonlinearity and modulator imperfections in dual-band concurrent transmitter using neural networks," Electron. Lett., vol. 49, no. 4, pp. 253-255, Feb. 2013
[24] Y.-J. Liu, W. Chen, J. Zhou, B. H. Zhou, and Y. N. Liu, "Joint predistortion of IQ impairments and PA nonlinearity in concurrent dualband transmitters," in Proc. 42nd Eur. Microw. Conf., Oct. 2012, pp. $132-135$.

[25] M. Younes and F. M. Ghannouchi, "On the modeling and linearization of a concurrent dual-band transmitter exhibiting nonlinear distortion and hardware impairments," IEEE Trans. Circuits Syst. I, Reg. Papers, vol. 60, no. 11, pp. 3055-3068, Nov. 2013.

[26] R. Wang, Y. K. Su, and C. H. Liu, "3-5 GHz cascoded UWB power amplifier," in IEEE Asia-Pacific Circuits Syst. Conf., Dec. 2006, pp. 367-369.

[27] M. Rawat, P. Roblin, C. Quindroit, N. Narharishetti, R. Pond, K. Salam, and C. Xie, "Characterization and modeling scheme for harmonics at power amplifier output," in 83 rd ARFTG Microw. Meas. Symp., Tampa, FL, USA, Jun. 2014, pp. 1-4.

[28] M. Rawat, P. Roblin, C. Quindroit, K. Salam, and C. Xie, "Digitally supported feed-forward harmonic cancellation for filter-less ultra-wideband transmitters," in IEEE Int. Microw. RF Conf., Bangalore, India, Dec. 2014, pp. 1-4.

[29] M. Omer, R. Rimini, P. Draxler, and J. S. Kenney, "Interference cancellation for odd harmonics of envelope tracking RF power amplifier systems," in IEEE MTT-S Int. Microw. Symp. Dig., Jun. 2013, pp. 1-3.

[30] D. E. Root, J. Verspecht, D. Sharrit, J. Wood, and A. Cognata, "Broadband polyharmonic distortion (PHD) behavioral models from fast automated simulations and large-signal scattering functions," IEEE Trans. Microw. Theory Techn., vol. 53, no. 11, pp. 3656-3664, Nov. 2005.

[31] P. J. Tasker and J. Benedikt, "Waveform inspired models and the harmonic balance emulator," IEEE Microw. Mag., vol. 12, no. 2, pp. $38-54$, Apr. 2011

[32] J. Sombrin, G. Soubercaze-Pun, and I. Albert, "Discontinuity at origin in Volterra and band-pass limited models," in IEEE MTT-S Int. Microw. Symp. Dig., Jun. 2013, pp. 1-4.

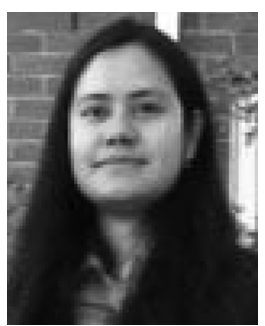

Meenakshi Rawat (M'09) received the B.Tech. degree in electrical engineering from the Govind $\mathrm{Bal}-$ labh Pant University of Agriculture and Technology, Uttarakhand, India, in 2006, and the M.Sc. and Ph.D. degrees in electrical and computer engineering from the University of Calgary, Calgary, $\mathrm{AB}$, Canada, in 2012.

From September 2012 to June 2013, she was a Post-Doctoral Research Fellow with the University of Calgary. From July 2013 to June 2014, she was a Post-Doctoral Project Researcher/Scientist with The Ohio State University. She is currently an Assistant Professor with the Indian Institute of Technology (IIT), Roorkee, India.

Dr. Rawat was workshop co-chair for ARFTG-82 and session co-chair for MMwave and THz Designs for iMARC 2014, Bangalore, India. She was part of the Calgary group that won Overall Championship and Best Design Prize of the 3rd Annual Smart Radio Challenge, Wireless Innovation Forum. She was also the three-time recipient of the Research Production Award of the University of Calgary and Best Poster Award of the 82nd Automatic RF Techniques Group (ARFTG) Conference, Columbus, OH, USA, in 2013.

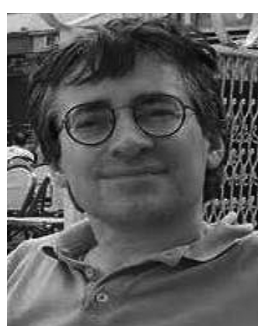

Patrick Roblin (M'85-SM'14) was born in Paris, France, in September 1958. He received the Maitrise de Physics degree from the Louis Pasteur University, Strasbourg, France, in 1980, and the M.S. and D.Sc. degrees in electrical engineering from Washington University, St. Louis, MO, USA, in 1982 and 1984, respectively.

In 1984, he joined the Department of Electrical Engineering, The Ohio State University (OSU), Columbus, $\mathrm{OH}$, USA, as an Assistant Professor, and is currently a Professor. His current research interests include the measurement, modeling, design, and linearization of nonlinear $\mathrm{RF}$ devices and circuits such as oscillators, mixers, and power amplifiers. $\mathrm{He}$ is the Founder of the NonLinear RF Research Laboratory, OSU. With OSU, he has developed two educational RF/microwave laboratories and associated curriculum for training both undergraduate and graduate students. He is lead author of two textbooks, High-Speed Heterostructure and Devices (Cambridge Univ. Press, 2002) and Nonlinear RF Circuits and Nonlinear Vector Network Analyzers (Cambridge Univ. Press, 2011). 


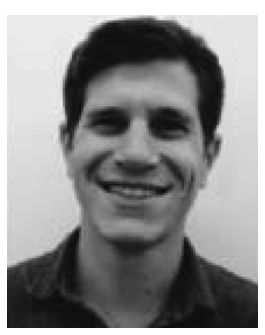

Christophe Quindroit was born in Corbeil-Essonnes, France, in October 1982. He received the M.Tech. and M.S. degrees in electronics from the Ecole Polytechnique de l'Universit de Nantes, Nantes, France, in 2005, and the Ph.D. degree in electronics from XLIM, University of Limoges, Limoges, France, in 2010.

He was a Project Engineer with Alcatel-Lucent, Lannion, France. He was a Research Engineer with The Ohio State University, Columbus, OH, USA. He is currently a Radio Engineer with LPA Concepts, Martillac, France. His research interests include analog system-level modeling, power amplifier (PA) linearization techniques, and field-programmable gatearray (FPGA) implementation.

Khan Salam (M'02), photograph and biography not available at the time of publication.

Chenggang Xie (M'96), photograph and biography not available at the time of publication. 